Utah State University

DigitalCommons@USU

\title{
Expert vs. Novice: Problem Decomposition/Recomposition in
} Engineering Design

\author{
Ting Song \\ Utah State University
}

Follow this and additional works at: https://digitalcommons.usu.edu/etd

Part of the Engineering Education Commons

\section{Recommended Citation}

Song, Ting, "Expert vs. Novice: Problem Decomposition/Recomposition in Engineering Design" (2014). All Graduate Theses and Dissertations. 3104.

https://digitalcommons.usu.edu/etd/3104

This Dissertation is brought to you for free and open access by the Graduate Studies at DigitalCommons@USU. It has been accepted for inclusion in All Graduate Theses and Dissertations by an authorized administrator of DigitalCommons@USU. For more information, please contact digitalcommons@usu.edu.

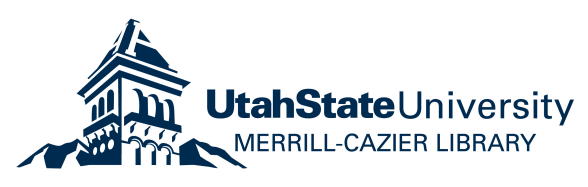


EXPERT VS. NOVICE: PROBLEM DECOMPOSITION/RECOMPOSITION IN

\section{ENGINEERING DESIGN}

by

\section{Ting Song}

A dissertation submitted in partial fulfillment

of the requirements for the degree

of

DOCTOR OF PHILOSOPHY

in

\section{Engineering Education}

Approved:

Kurt H. Becker, PhD

Major Professor

John S. Gero, PhD

Committee Member

Edward M. Reeve, PhD

Committee Member
M. Scott DeBerard, PhD

Committee Member

Oenardi Lawanto, PhD

Committee Member

Mark R. McLellan, PhD

Vice President for Research and

Dean of the School of Graduate

Studies

UTAH STATE UNIVERSITY

Logan, Utah 
Copyright (C) Ting Song 2014

All Rights Reserved 


\begin{abstract}
Expert vs. Novice: Problem Decomposition/Recomposition in Engineering Design by
\end{abstract}

Ting Song, Doctor of Philosophy

Utah State University, 2014

\author{
Major Professor: Dr. Kurt Becker \\ Department: Engineering Education
}

The purpose of this research was to investigate the differences of using problem decomposition and problem recomposition among dyads of engineering experts, dyads of engineering seniors, and dyads of engineering freshmen. Fifty participants took part in this study. Ten were engineering design experts, 20 were engineering seniors, and 20 were engineering freshmen. Participants worked in dyads to complete an engineering design challenge within an hour. The entire design process was video and audio recorded. After the design session, members participated in a group interview.

This study used protocol analysis as the methodology. Video and audio data were transcribed, segmented, and coded. Two coding systems including the FBS ontology and "levels of the problem" were used in this study. A series of statistical techniques were 
used to analyze data. Interview data and participants' design sketches also worked as supplemental data to help answer the research questions.

By analyzing the quantitative and qualitative data, it was found that students used less problem decomposition and problem recomposoition than engineer experts in engineering design. This result implies that engineering education should place more importance on teaching problem decomposition and problem recomposition. Students were found to spend less cognitive effort when considering the problem as a whole and interactions between subsystems than engineer experts. In addition, students were also found to spend more cognitive effort when considering details of subsystems. These results showed that students tended to use dept-first decomposition and experts tended to use breadth-first decomposition in engineering design. The use of Function (F), Behavior (B), and Structure (S) among engineering experts, engineering seniors, and engineering freshmen was compared on three levels. Level 1 represents designers consider the problem as an integral whole, Level 2 represents designers consider interactions between subsystems, and Level 3 represents designers consider details of subsystems. The results showed that students used more $\mathrm{S}$ on Level 1 and 3 but they used less F on Level 1 than engineering experts. The results imply that engineering curriculum should improve the 
teaching of problem definition in engineering design because students need to understand the problem before solving it. 


\section{PUBLIC ABSTRACT}

Expert vs. Novice: Problem Decomposition/Recomposition in Engineering Design by

Ting Song, Doctor of Philosophy

Utah State University, 2014

The purpose of this research was to investigate the design behavior. A commonly used design strategy called "problem decomposition and recomposition" was the focus of the study. Dyads of engineering experts, dyads of engineering seniors, and dyads of engineering freshmen completed an engineering design challenge within an hour. The entire design process was video and audio recorded. After the design session, participants participated in a group interview.

The conversation and behavior from the design were transcribed, segmented, and coded. Two coding systems including the FBS ontology and "levels of the problem" were used in this study. A series of statistical techniques were used to analyze data. Interview data and participants' design sketches also worked as supplemental data to help answer the research questions.

There were a series of interesting findings that emerged from analyzing the quantitative and qualitative data. Several gaps were identified between students' design behaviors and experts'. The findings of this study will help better understand the development of expertise in engineering design and lead to improvements in engineering education. 


\section{ACKNOWLEDGEMENTS}

I would like to thank my advisor, Dr. Kurt Becker. Dr. Becker has been helping me through my entire master and doctoral programs. I have made great progress as a researcher under Dr. Becker's tutelage. I also want to thank my committee members, Drs. Scott DeBerard, John Gero, Oenardi Lawanto, and Edward Reeve. Their feedback and critiques have been extremely helpful to me and my research.

Additional thanks should go to my amazing friends who have made my life so colorful and fun. I especially want to thank Justin Kartchner who helped me recruit four engineers to participate in my study.

Last, but definitely not least, I would like to thank my family. My grandma, who passed away in 2012, has inspired me to be a more kindhearted person. She will be always with me. I want to thank my parents, who have been so supportive to whatever decisions I made in my life. I want to thank my husband, who is the love of my life, for making me a happier person than I ever could be. I love you! 
$\begin{array}{lll} & \text { Page }\end{array}$

ABSTRACT ........................................................................................

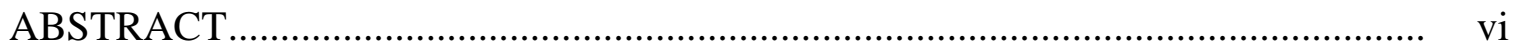

LIST OF TABLES ..................................................................................

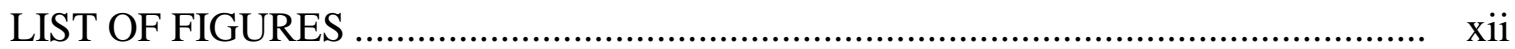

\section{CHAPTER}

I. INTRODUCTION ........................................................................ 1

Purpose Statement................................................................................. 5

Research Questions .............................................................................. 5

Definition of Terms......................................................................... 6

Limitations ............................................................................. 8

Assumptions ..................................................................................... 9

Significant of the Study .................................................................. 9

II. LITERATURE REVIEW .......................................................... 12

Overview and Selection Criteria ........................................................... 12

Engineering Design........................................................................ 14

Engineering Design Challenge .......................................................... 17

FBS Framework ....................................................................... 20

Problem Decomposition/Recomposition ................................................ 23

Expert-Novice ................................................................................. 27

Protocol Analysis ..................................................................................... $\quad 30$

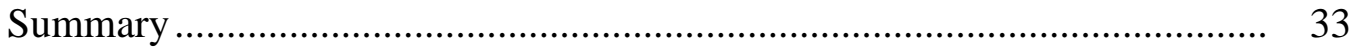


III. METHODOLOGY ..................................................................... 34

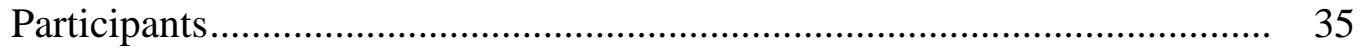

Participant Selection ..................................................................... 36

Institutional Review Board .................................................................. 38

Data Collection ........................................................................... 39

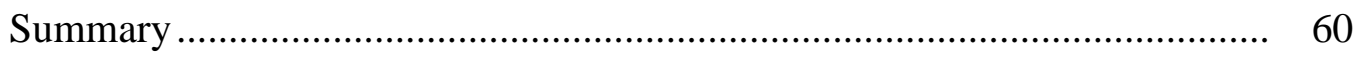

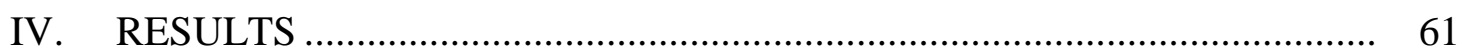

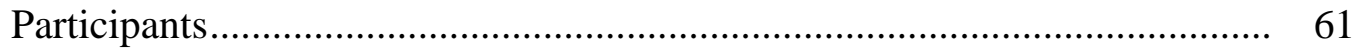

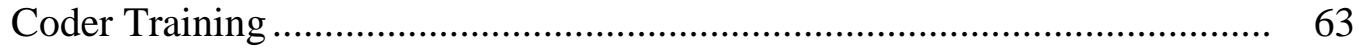

Research Question 1 .......................................................................... 68

Research Question 2 ................................................................ 73

Research Question 3 ........................................................................... 80

Other Themes and Phenomenon Found from Qualitative Data................... 86

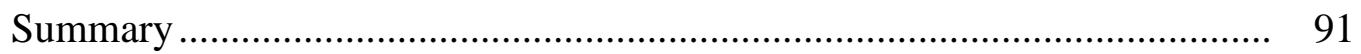

V. DISCUSSIONS, IMPLICATIONS, AND RECOMMENDATIONS ........... 92

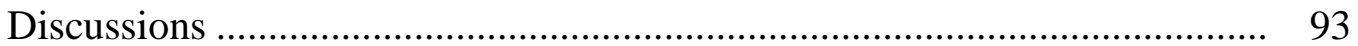

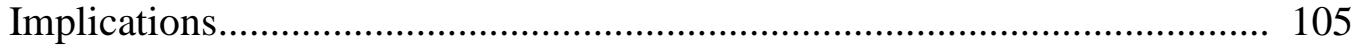

Recommendations for Future Study ..................................................... 110

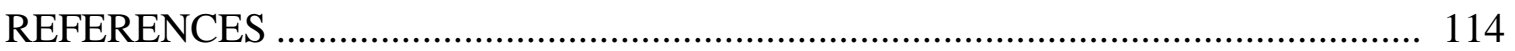

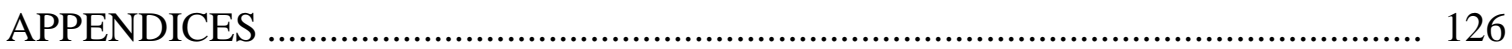

Appendix A:Visual Map of Literatures ............................................ 127

Appendix B:Double-Hung Window Opener Design............................... 129

Appendix C: Interview Questions ....................................................... 131 
Appendix D: Anonymous Demographic Questionnaire for Students......... 133

Appendix E: Anonymous Demographic Questionnaire for Engineers ....... 135

Appendix F: Informed Consent Form for Students ............................... 137

Appendix G:Informed Consent Form for Engineers .............................. 141 


\section{LIST OF TABLES}

Table

1. Levels of the Problem.

2. Example of FBS Codes and Arbitrated Codes

3. Sample Codes for Levels of the Problem

4. Calculation of Percentages of Codes for FBS ontology

5. Calculation of Percentages of Codes for Levels of the Problem

6. Example of Problem Decomposition and Problem Recomposition

7. Example of Qualitative Data Analysis

8. Means and Standard Deviations of Problem Decomposition and Problem

Recomposition

9. Results of Research Question 1 .

10. Means and Standard Deviations of Levels of the Problem

11. Results of Research Question 2 .

12. Means and Standard Deviations of FBS on Different Levels of the Problem ....

13. Dyad Comparisons (p-value) of FBS on Level 1

14. Dyad Comparisons ( $\mathrm{p}$-value) of FBS on Level 2

15. Dyad Comparisons (p-value) of FBS on Level 3 


\section{LIST OF FIGURES}

Figure

Page

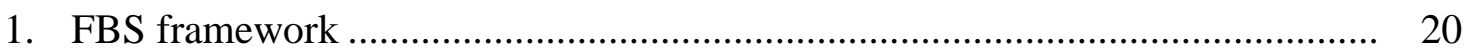

2. An example of a cell phone (picture resource: Dr. John Gero) ......................... 22 


\section{CHAPTER I}

\section{INTRODUCTION}

Design is recognized as the critical element of engineering thinking which differentiates engineering from other problem solving approaches (Dym, Agogino, Eris,

Frey, \& Leifer, 2005). One of the primary goals of engineering design education is to equip students with the capability to become expert design engineers. To develop this capability in students, educators require a detailed knowledge of the cognitive behavior of both undergraduate students and expert design engineers. However, there is insufficient information known about the cognitive behavior of expert design engineers, since most studies are focused on individual student engineers or early professional engineers. While the significant differences between expert behaviors and novice behaviors have been studied heavily in many other STEM fields ranging from biology to medicine, it has been notably understudied in engineering.

Today, the American engineering education system should provide high-quality education to help students compete in the future job market. Students need to learn new technologies and understand social and global issues related to their fields. "In an increasingly global marketplace, our graduates are and will be expected to work on multinational teams, have a global perspective, and be culturally and linguistically literate. 
They must possess communication skills to interact effectively in the community and within the professional and political arenas" (American Society for Engineering Education, 2004, p. 2).

Among the engineering skills required, engineering design is fundamental for engineering graduates because engineering design is a major task used by practicing engineers. There are various definitions of engineering design and according to the Accreditation Board for Engineering and Technology (ABET), engineering design is ...the process of devising a system, component, or process to meet desired needs. It is a decision-making process (often iterative), in which the basic sciences, mathematics, and the engineering sciences are applied to convert resources optimally to meet these stated needs. (ABET, 2007, p.3)

Engineering design is open-ended and requires designers to be creative. There is a vital need for engineering graduates who are able to design effective solutions to meet customers' various requirements (Sheppard, 2003). Despite its importance, today, there are some limitations in teaching engineering design (de Vere, Melles, \& Kapoor, 2010). There is a gap between skills developed at universities and skills needed in the industry (Jonassen, Strobel, \& Lee, 2006; Patil, 2005). Engineering education teaches students more scientific and analytical skills than abilities to execute finished products to satisfy 
clients' needs (Dym, 1999; Nair, Mertova, \& Patil, 2009). Students usually spend their

first and second years learning foundational courses that are often taught by mathematics and science teachers who have few experiences in engineering (Dym, 2004). In contrast, they spend little time with design courses. As a result, according to Nair et al. (2009), they frequently fail to survive in the industry. Engineering curriculum needs a major overhaul to prepare students with practical skills and knowledge (Kirschenman \& Brenner, 2011) because "the 'real world' provides the materials and devices with which engineers design, and the world is where the performance of systems is verified" (Moore \& Voltmer, 2003, p. 454).

The use of design strategies plays a significant role in engineering design, and a commonly used strategy is problem decomposition/recomposition. It is frequently used by experienced engineers, especially for solving complex engineering problems (Vincenti, 1990). The process of problem decomposition involves breaking the design problem into smaller independent sub-problems (Arvanitis, Todd, Gibb, \& Orihashi, 2001). Each subproblem can be further broken into even smaller problems (Arvanitis et al., 2001) and the decomposition process stops when designers can directly approach each sub-problem. The problem recomposition is a bottom-up process that usually comes with problem decomposition. It is a process of recomposing all sub-solutions (Chandrasekaran, 1990) 
in the premise of satisfying requirements of the combining design (Hall, Jackson, Lanney, Nuseibeh, \& Rapanotti, 2002). Instead of focusing on a complex design problem as a whole, engineers can work on several smaller, more approachable sub-problems using this process, which makes the process of engineering design more efficient. According to Ball, Evans, and Dennis (1994) and Ho (2001), the use of problem decomposition/recomposition is developed through the accumulation of experiences because it is rarely taught in engineering institutions. Studies have identified a gap between engineering novices and engineering experts when it comes to problem decomposition/recomposition skills in engineering design (Ball et al., 1994; Ho, 2001; McCracken, 1997).

In order to improve the quality of engineering education and prepare students for real world challenges, schools need to teach students "expert-like" skills. To reach this goal, more research needs to be conducted to study engineering design skills in a realworld environment solving actual problems.

Currently, studies on this topic are rarely found. To the extent that past works are available (Ball et al., 1994; Ho, 2001; McCracken, 1997), most studies about problem decomposition/recomposition have focused on individuals instead of dyads. It is valuable to study problem decomposition and recomposition in the context of dyads because in the 
real world engineers usually work in dyads to solve engineering problems. By

investigating this topic in the context of collaborative engineering design, researchers will have a better understanding of the development of expertise and the use of problem decomposition/recomposition in practical settings.

\section{Purpose Statement}

The focus of the study is to compare the use of problem

decomposition/recomposition between experts and novices during engineering design.

Dyads of expert engineers were compared to dyads of engineering freshmen students and engineering senior students while completing an engineering design challenge.

\section{Research Questions}

The broad research question for this study is: In the process of engineering design, how do experts approach the design problem differently from engineering students? More specifically, there were three sub-research questions guiding this study:

1. Do engineering experts use problem decomposition and recomposition more than engineering seniors and engineering freshmen in engineering design?

2. Which level of the problem do engineering experts, seniors, and freshmen spend most cognitive effort on? 
3. What mental activities are used by engineering experts, seniors, and freshmen when they approach different levels of the problem?

\section{Definition of Terms}

Cognition: Thinking skills and thinking processes used in problem solving and

learning (Marzano et al., 1988).

Cognitive effort: The amount of thinking skills and thinking processes spent by designers to solve design problems.

Decomposition: Decomposition is the process of breaking a problem down into individual, manageable sub-problems (Arvanitis et al., 2001).

Design: It is a "systematic, intelligent process in which designers generate, evaluate, and specify concepts for devices, systems, or processes whose form and function achieve the clients' objectives or users' needs, while satisfying a specified set of constraints" (Dym et al., 2005, p. 103).

Engineering Design: It is “...the process of devising a system, component, or process to meet desired needs. It is a decision-making process (often iterative), in which the basic sciences, mathematics, and the engineering sciences are applied to convert resources optimally to meet these stated needs" (ABET, 2007, p. 3). 
Engineering design challenge: It is a group based activity in which participants engage in solving a real world open-ended problem using engineering method. Solutions generated are test and analyzed. Final design decision is made according to the result of test and analysis (Lammi, 2011).

Function-Behavior-Structure (FBS) framework: FBS framework was developed by Gero (Gero, 1990; Gero \& Kannengiesser, 2004; Gero \& Mc Neill, 2006). The basic concept of this framework is the classification which describes three aspects of the design process: Function, Behavior, and Structure. Function $(\mathrm{F})$ variables describe the teleology of the object (what it is for). Behavior (B) variables describe the attributes that are derived or expected to be derived from the structure $(\mathrm{S})$ variables of the object (what it is does). Structure (S) variables describe the components of the object and their relationships (what it is) (Gero \& Kannengiesser, 2004, p. 374). Behavior includes behavior derived from the structure $(\mathrm{Bs})$ and expected from the structure $(\mathrm{Be})$ (Gero \& Kannengiesser, 2004). R represents requirements derived from clients and D represents design descriptions (Gero \& Kannengiesser, 2007). The design actually is a consequence of a series of processes among above FBS variables.

Mental Activity: The concept of intellectual processes that involve cognitive engagements. 
Problem decomposition: It is a top-down process which breaks the problem into smaller sub-problems. Each sub-problem can be further broken into even smaller problems (Arvanitis et al., 2001). The breaking process stops when designers can directly approach sub-problems.

Problem recomposition: It is a bottom-up process that usually comes with problem decomposition. It is a process of recomposing sub-solutions (Chandrasekaran, 1990). In this process, sub-solutions are not simply combined but recomposed in a way satisfying the combined requirements (Hall et al., 2002).

Protocol Analysis: Protocol analysis is a methodology for capturing subjects' thoughts by eliciting their verbal reports (Williams, Gero, Lee, \& Paretti, 2011).

\section{Limitations}

The study had the following limitations:

1. The project was a pilot study. The data of the study were collected from a small sample of college engineering freshmen, engineering seniors, and practicing engineers.

2. The study only investigated the engineering design process through the "design proposal" stage. Participants in this study did not build, test, and evaluate their design. 
3. Verbal protocol offered partial information of how designers think during the design process. It did not record the process of thinking completely.

4. The engineering design challenge was recommended to complete in an hour. It did not totally generalize engineering design as the process typically requires for days or weeks to finish.

5. The design task used in this study is not an authentic design task in academic setting.

\section{Assumptions}

The study made the following assumptions.

1. Practicing engineers were more skilled in engineering design than college engineering freshmen and seniors.

2. The instrument used in this study accurately collected data from participants.

3. The presence of researchers did not affect the performance of participants.

\section{Significances of the Study}

This study contributed to the body of research related to engineering expertise. By comparing the process of problem decomposition and problem recomposition among dyads of engineering freshmen, dyads of engineering seniors, and dyads of engineering 
experts, this study helped better understand the characteristics of expertise in engineering design.

The direct significance of the study included: quantitatively characterizing the design thinking of both engineering students and expert engineers from a design cognition perspective; measuring differences between the design cognition of engineering students and expert engineers; adding to the knowledge of the development of design thinking across education and experience; and proposing educational interventions that narrow the differences between students and experts.

Equally important, by applying a cognitively based measurement technique for studying design thinking, this study contributed to the development of quantitative measurements of the effects of educational interventions and of experience. In addition, the findings of this study helped engineering educators improve the curriculum of engineering design. New teaching strategies will be developed in order to teach students expert-like skills, which will contribute to the improvement of college engineering education.

In addition, by measuring the differences between the design cognition of engineering students and expert engineers, we can better understand novice/expert continuum in engineering design. By quantitatively characterizing the design thinking of 
both engineering students and expert engineers from a design cognition perspective we can develop novel pathways to engineering education. By indentifying gaps between student and expert design behavior we can move toward interventions in teaching to reduce gaps. By identifying new ways engineering education models evolve we can add to the knowledge of design thinking across education and experience, and present knowledge that can be used to develop educational interventions that move students along a trajectory towards expert design behavior. 
CHAPTER II

\section{LITERATURE REVIEW}

\section{Overview and Selection Criteria}

Engineering design is an important focus of engineering education and a major

activity for practicing engineers. Studies have shown that there is a gap between skills

students learned in school and those required in the industry (Nair et al., 2009). The

teaching of design needs improvements (McMasters, 2004; Todd \& Magleby, 2004) and

as previously stated, problem decomposition/recomposition is a glaring omission in

engineering education. The study addressed this problem by investigating the differences

between engineering freshman dyads, engineering senior dyads and engineering expert

dyads. The focus of the study was the practice of problem decomposition and

recomposition during the engineering design process.

To develop a better understanding of existing research and the topic at hand, this

study reviewed six interrelated areas. The six areas included: Engineering Design, Expert

vs. Novice, Engineering Design Challenges, FBS Framework, Problem

Decomposition/Recomposition, and Protocol Analysis. Their relationships are shown in

the visual map in Appendix A. 
In the Engineering Design section, research related to engineering design was examined to help set the context of this study. Expert vs. Novice is a commonly used technique in studying expertise in various fields and in the Expert vs. Novice section, the focus was the development of expertise and how this technique was used in studying engineering design. Engineering design challenges have been widely used in engineering curriculum and engineering research. The review of literature in this section focused on how the technique was used in teaching and research. FBS Framework was developed by Gero and colleagues (Gero, 1990; Gero \& Kannengiesser, 2004) and was considered an effective tool to investigate the design process. Studies on the validity of this tool were reviewed in this section. Problem decomposition/recomposition as a widely used design strategy was examined to understand its application by engineering novices and experts. Protocol analysis is a method used to document the process of design. The validity of this method was investigated in the Protocol Analysis section.

A body of literature was established to understand the existing studies in engineering education. This study reviewed literatures from a number of sources. Most of the literature was from Journal of Engineering Education, Journal of Industrial Teacher Education, Research in Engineering Design, Design Studies, European Journal of 
Engineering Education, and the International Journal of Technology and Design

Education. Some of the literature was from other resources that were not listed above.

The following searching engines and database were used to search the literature of this study: Google Scholar, Education Full Text via EBSCO Host, Education Resources Information Center (ERIC) via EBSCO Host, ERIC via the US Department of Education, Professional Development Collection, Digest of Education Statistics, and Digital Dissertations. In addition, Utah State University Merrill-Cazier Library was also an important resource to search for the literature.

In the process of literature review, the following criteria were applied: (a) peer reviewed, and (b) addresses the issue of engineering education. The following key words or a combination of them were used to search the literature: engineering design, engineering education, design challenge, group study, expertise in engineering, FBS, protocol analysis, and problem decomposition/recomposition.

\section{Engineering Design}

Design is a creative, open-ended, and experiential process that aims at problem solving. Engineering design as an important branch of design, is a "systematic, intelligent process in which designers generate, evaluate, and specify concepts for devices, systems, or processes whose form and function achieve the clients' objectives or users' needs, 
while satisfying a specified set of constraints" (Dym et al., 2005, p.103). Engineering

design is a central part of engineering education because it is considered a distinguishing activity of engineers. For this reason, it has been emphasized as a focus for engineering education for several decades (Dym et al., 2005).

Engineering design contains multiple interconnected variables and in general, these variables belong to two types: technical variables and non-technical variables. Technical variables include time, power, weight, height, and so forth; non-technical variables include cost, reliability, safety, and so forth. These variables have complex interrelationships and often vary non-linearly along some scale. "Aggregate behavior is qualitatively distinct from the sum of behaviors of individual components and indicates a complex engineered system, such as highways, the Internet, the power grid, and many others, which are all around us" (Katehi, Pearson, Feder, \& NRC, 2009, p. 125).

Because of the complexity and ill-structure of engineering design, no model can perfectly represent its process (Mawson, 2003). Still, researchers have developed various engineering design models to approximate the process (Atman et al., 2007; Cross, 2004; Draper, 2009; Dym, Little, Orwin, \& Spjut, 2009; Eide, Jenison, Mashaw, \& Northrup, 2002; Hailey, Erekson, Becker, \& Thomas, 2005). Dym and colleagues (Dym et al., 2009) developed an engineering design model comprising 5steps: problem definition, 
conceptual design, preliminary design, detailed design, and design communication. The seemly simple model is complicated by the sub-steps in each step. For example, the six sub-steps of conceptual design include establish functions, establish requirements, establish means for functions, generate design alternatives, refine and apply metrics to design alternatives, and choose a design (Dym et al, 2009, p.25). Eide and colleagues (Eide et al., 2002) defined the engineering design process as 10 steps: identification of a need, problem definition, search, constraints, criteria, alternative solutions, analysis, decision, specification, and communication. The Dym and Eide models have been widely used in teaching the engineering design process. Pahl and his colleagues' book (Pahl et al., 2007) has been widely used as an engineering textbook. In the book, they explained engineering design as the following aspects: product planning, solution finding, evaluation, product development, task clarification, conceptual design, embodiment design, mechanical ranges, mechatronics, size ranges, modular products, design quality and design for minimum cost.

In the process of reviewing various engineering design models (Atman et al., 2007; Cross, 2004; Draper, 2009; Dym et al., 2009; Eide et al., 2002; Hailey et al., 2005), a few steps are found to be commonly used, such as definition of the problem, establishing the objectives and criteria, alternative solutions, and optimization. Although 
models reviewed were presented in linear format, the process of engineering design is iterative to gain better results (Dym et al., 2009). As noted by Sheppard, Macatangay, Colby, and Sullivan (2009), "[engineering design] is not linear: at any phrase of the (engineering design) process, the engineer may need to identify and define subproblems, then generate and evaluate solutions of subproblems to integrate back into the overall process" (p. 104). Other than the common steps mentioned in various design models, successful engineering design require skills such as teamwork, communication, and time management as well (Sheppard et al., 2009). Engineering design is often taught using an engineering design challenge to reinforce to students the various design models.

\section{Engineering Design Challenge}

Terms used to describe engineering design challenge are different from study to study. They refer to similar pedagogical approaches in teaching, which enable students to learn knowledge in the process of solving engineering problems related to their life. These terms include challenge-based instruction (Roselli \& Brophy, 2006), active learning (Yeager, 2002), design challenge (Romero, Slater, \& DeCristofano, 2006), project-based learning (Dym et al., 2005), etc.

Engineering design challenges are widely and effectively used in teaching engineering and engineering education research. Engineering design challenge can be 
used in formal academic circumstances, in informal settings, and even in service learning activities (O'Neil \& Lima, 2003). Dym and colleagues (2005) believed that engineering design challenges could benefit student learning from many aspects.

In theory, these challenges should include the entire engineering design process, but practical engineering design challenges are extremely complex and ill-structured.

Engineers have to deal with ambiguous specifications of goals, many degrees of freedom in the problem statement, multiple solutions (Goel \& Pirolli, 1989), which requires integrating multiple knowledge domains to complete the design challenge (Jonassen, 2000).That is the reason why in classrooms educators sometimes incorporate only parts of the design process according to the objective and the length of the curriculum (Atman et al., 2007; Katehi et al., 2009).

There are a number of engineering design challenges which vary in duration and topic. Researchers should select engineering design challenge according to the research question. An appropriate design challenge ensures the success of the study. A widely used design challenge is the window opener. To complete this design challenge, participants need to design a double-hung window opener that assists the elderly with raising and lowering windows. This challenge has been used by Gero (2010b) and other researchers (Williams et al., 2011) to study engineering design. Another widely used 
challenge is the playground design, where participants are asked to design a playground for residents in a mid-sized city. This design challenge has been used by Atman and colleagues (Atman \& Bursic, 1998; Atman et al., 2007) to study the engineering design process.

The above two design challenges have been extensively used in studies involving high school students and college freshmen students (Atman, Kilgore, \& McKenna, 2008; Gero, 2010). The reason for that is completing engineering design challenges requires applying mathematical, scientific, and technological theories. Marin, Armstrong, and Kays (1999) stated: "students must first learn to crawl before they can walk or run. This means there must be sufficient course work in the appropriate engineering science upon which the capstone design experience will be built" (p. 19). Students in high school and college freshman do not have enough related knowledge to solve complex design

problems and the window opener challenge and the playground challenge are appropriate for their knowledge level.

\section{FBS Framework}

Function-Behavior-Structure (FBS) ontology is used to classify design processes and it was developed by Gero (Gero, 1990; Gero \& Kannengiesser, 2004; Gero \& Mc Neill, 1998). The FBS ontology enables researchers to compare the design cognition in 
various situations, including different designers, different design tasks, different design requirements, and different design time. The FBS ontology provides a set of irreducible foundational concepts of design and designing, which covers the acts of designing and the representation of the design. The definition and conceptualization of the ontology is illustrated in Figure 1.

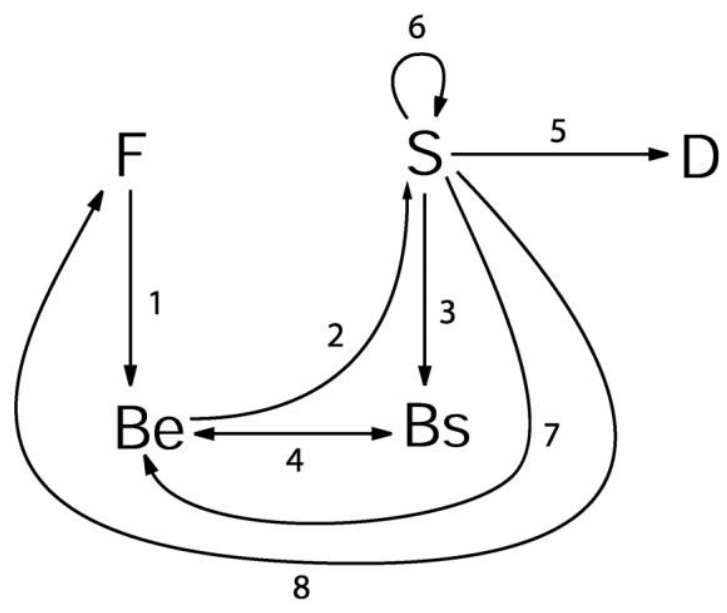

Figure 1. FBS framework (Resource: Gero, Kan, \& Pourmohamadi, 2011).

The basic concept of this framework is the classification which describes three aspects of the design process: Function (F) variables describe the teleology of the object; Behavior (B) variables describe the attributes that are derived or expected to be derived from the structure $(S)$ variables of the object; and Structure $(S)$ variables: describe the components of the object and their relationships (Gero \& Kannengiesser, 2004, p. 374). 
Behavior includes behavior derived from the structure (Bs) and expected from the structure (Be) (Gero \& Kannengiesser, 2004). R represents requirements derived from clients and D represents design descriptions (Gero \& Kannengiesser, 2007). The design actually is a consequence of a series of processes among above FBS variables:

1. Formulation (process 1) transforms the design requirements, expressed in function $(\mathrm{F})$, into behavior $(\mathrm{Be})$ that is expected to enable this function.

2. Synthesis (process 2) transforms the expected behavior (Be) into a solution structure (S) that is intended to exhibit this desired behavior.

3. Analysis (process 3) derives the "actual" behavior (Bs) from the synthesized structure (S).

4. Evaluation (process 4) compares the behavior derived from structure (Bs) with the expected behavior to prepare the decision if the design solution is to be accepted.

5. Documentation (process 5) produces the design description (D) for constructing or manufacturing the product.

6. Reformulation type 1 (process 6) addresses changes in the design state space in terms of structure variables or ranges of values for them if the actual behavior is evaluated to be unsatisfactory. 
7. Reformulation type 2 (process 7 ) addresses changes in the design state space in terms of behavior variables or ranges of values for them if the actual behavior is evaluated to be unsatisfactory.

8. Reformulation type 3 (process 8) addresses changes in the design state space in terms of function variables or ranges of values for them if the actual behavior is evaluated to be unsatisfactory. (Gero \& Kannengiesser, 2007, p.3)

An example of FBS ontology is shown in Figure 2. The requirements are expressed by F. Be expressed how to enable the function. S represents the structure of the design.

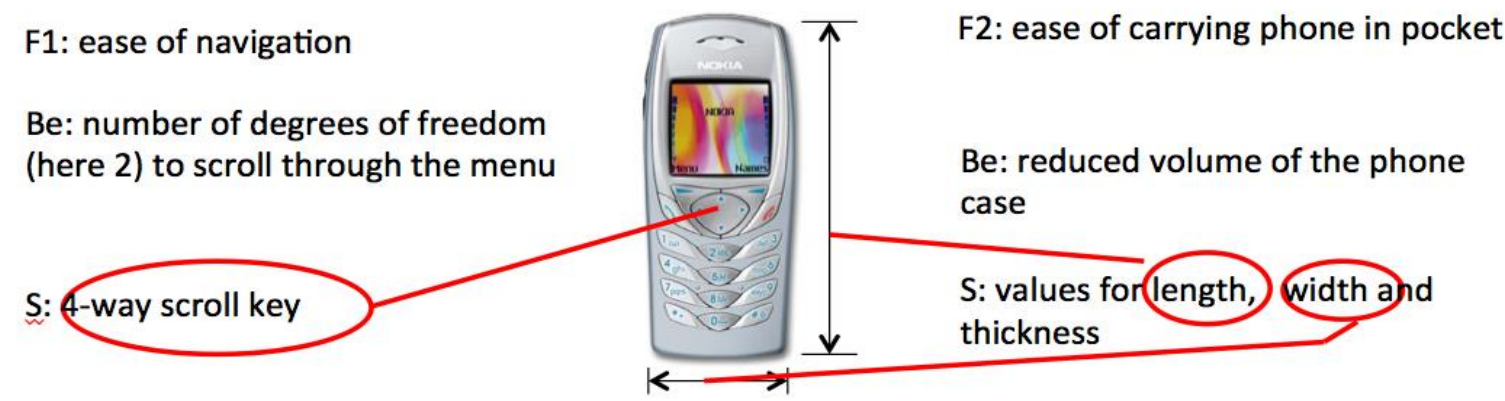

Figure 2. Example of a cell phone (picture resource: Dr. John Gero's training session of FBS ontology, July, 2012).

FBS framework became widely used and discussed (Vermaas \& Dorst, 2007) in studying design (Alexiou, 2010; Hmelo-Silver, 2004) and engineering design (Ho, 2001; Mulet \& Vidal, 2008), because 
(FBS) lies specifically in its move from the analysis of design behaviors, which may be tied to a single domain, to the elucidation of design cognition; this move is critical to furthering the ability of design researchers to make robust comparisions across a wide variety of settings. (Williams et al., 2011, p. 2)

\section{Problem Decomposition/Recomposition}

In the world of design, there are three types of design decomposition, including product decomposition, problem decomposition and process decomposition (Kusiak \& Larson, 1995).

......product decomposition is used to prescribe the physical elements of the product, namely, structural decomposition; problem decomposition is used to decompose a complex engineering problem into a manageable number of subproblems; process decomposition is used to decompose design activities in the design process. (Deng \& Zheng, 2006, p. 1106)

Problem decomposition and recomposition is a frequently used technique in

problem solving (Anderson, 1983). The process of problem decomposition is a top-down process. It breaks the problem into smaller sub-problems. Each sub-problem can be further broken into even smaller problems (Arvanitis et al., 2001). The breaking process stops when designers can directly approach sub-problems. The problem recomposition is 
a bottom-up process that usually comes with problem decomposition. It enables engineers to synthesize solutions to sub-problems together.

Akin's research (1986) started the cognitive basis of decomposition. He categorized considers two types of knowledge: representational knowledge and procedural knowledge (Akin, 1986). According to Akin (1986), representational knowledge is stored as hierarchical pieces. Those hierarchical pieces are utilized to establish a problem space to make problem decomposition happen.

On the other hand, some researchers believe that problem decomposition and recomposition operate "in an implicit or explicit manner" (Liikkanen \& Perttula, 2009). Explicit decomposition represents designer deliberately analyze a function structure in the early stage of the design process. Implicit decomposition refers that designers do not fully investigate function structure (Ho, 2001).

A similar concept of problem decomposition/recomposition is reverse engineering which is widely used in computer science industry (Marom et al., 2009). The first step in reverse engineering is "a decompositional analysis" (Schierwagen, 2012, p. 145) and a second step is "the (re-) construction of the original system" (Schierwagen, 2012, p. 145). Currently, reverse engineering is used as a "technology which generates a virtual 
representation of an existing part based on point data acquired through measuring techniques" (Vezzetti, 2009, p.780).

Previous studies identified two types of problem decomposition: breadth-first approach and depth-first approach. The breadth-first decomposition focuses on exploring various solutions of each sub-problem and avoids deep exploration to any specific solution in the early stage. On the other hand, depth-first decomposition tends to explore a specific sub-problem in detail before other sub-problems are investigated (Ormerod \& Ridgway, 1999). Marengo (1999) argued that the process of problem decomposition is related to the interdependencies in the problem. He believed that if the subparts of a problem have strong interdependencies then the problem cannot be decomposed. But Simon (1996) argued that when the solver has all the knowledge regarding the problem, any problem can be decomposed. One of the major findings in studying engineering design is that novices are prone to using depth-first decomposition in problem solving (Jeffries, Turner, Polson, \& Atwood, 1981) while experts are more inclined to use breadth-first decomposition to break the problem into "manageable chunk sizes" (Ormerod \& Ridgway, 1999, p. 402). This conclusion was confirmed by two experiments conducted by Ormerod and Ridgway (1999). Ho (2001) made a similar conclusion. In the study he investigated the engineering design process of a novice and an expert 
engineering designer. He found that by using problem decomposition/recomposition differently, two designers solved the problem in different ways: Experts tend to establish problem structure at the beginning in order to represent the problem in their own way..... While novice designers tend to eliminate the problem when they fail to handle it. They later on refine the new situation as a new problem at this initial state, and then approach the goal state of this new problem. (Ho, 2001, p.43)

Ball and colleagues (1994) had similar findings in their research in which the individual design behavior was studied in the context of electrical engineering.

The use of problem recomposition usually relies on problem decomposition and is a process of recomposing sub-solutions (Chandrasekaran, 1990). In this process, subsolutions are not simply combined but recomposed in a way satisfying the combined requirements (Hall et al., 2002). Unfortunately, there are few studies investigating the problem recomposition. In studies regarding problem decomposition and recomposition, researchers often focused on problem decomposition instead of recomposition. The reason is problem recomposition happens after problem decomposition and in most studies, novice participants struggled with problem decomposition so their use of problem recomposition could not be well studied. 
Problem decomposition/recomposition is widely used in solving engineering

problems, especially when problems are highly complex (Vincenti, 1990). This technique is especially important in software engineering because it is considered a critical cognitive skill in programming (Guindon, 1990).

Research showed that teaching problem decomposition/recomposition to novices might increase the efficiency of their design (Ho, 2001). However, engineering programs have not placed enough importance on teaching problem decomposition/recomposition. In the literature review, the researcher found few studies related to teaching this topic. Among the few studies in this area, Arvanitis and colleagues (2001) taught students to use problem decomposition in their classes but students failed to sufficiently apply this technique in problem solving. Problem decomposition/recomposition is a weak link in engineering education and should be considered a critical subject in teaching engineering design (Ho, 2001).

\section{Expert-novice}

Acquiring expertise is a complicated process. It involves not only the accumulation of practice and experience but also "qualitative shifts and stabilization in knowledge and performance" (Hoffman, 1996, p. 84). Experts organize their knowledge

more systematically than novices (Dufresne, Gerace, Hardiman, \& Mestre, 1992). 
Experts categorize information in meaningful chunks according to conceptual principles, while novices usually store their knowledge by superficial features (Egan \& Schwartz, 1979; Hoffman, 1996).

Many researchers study engineering design expertise via a number of different methodologies, and expert-novice continuum is a widely used one. By investigating the differences between experts and novices, researchers gained a deeper understanding of how to help students move from novices to expert designers.

Problem definition is the first and foundation-setting stage of engineering design. Novices are found to show many weaknesses in this stage. Experts tend to spend more time on this stage than novices (Atman et al., 2007). Novices do not spend enough time on problem definition because they do not realize that they do not completely understand the problem. Jain and Sobek (Jain \& Sobek, 2006) reached a similar conclusion in their research. Simply spending more time on problem definition does not improve the design (Yang, 2005). What novice designers need is to better understand the problem.

Gathering information is another important stage in engineering design (Jain \& Sobek, 2006), and experts usually acquire useful information more quickly. Christiaans and Dorst (1992) found that weak students who got stuck in information gathering either 
could not find enough useful information to move forward or gathered too much

information in the design process.

In the process of generating alternative solutions, experts tend to use balanced searching strategy (Fricke, 1996), which generates an appropriate number of solutions.

Unlike experts, novices are unable to adequately evaluate the potential of solutions. They usually generate either too few solutions or too many solutions. Both scenarios turned out to be ineffective design strategies. When few alternatives are available, novice designers tend to fixate on the solution stage too early. When there are too many alternative solutions, designers reach cognitive overload and tend to spend their time and energy on managing and organizing solutions instead of evaluating and improving solutions (Fricke, 1996).

In exploring solutions, novices are prone to use depth-first approach while experts are prone to use breadth-first approach (Cross, 2004). Depth-first approach is exploring a specific solution in depth and breadth-first approach is making preliminary evaluations of alternative solutions before exploring any of them in depth (Cross, 2004). A series of studies (Ahmed, Wallace, \& Blessing, 2003; Ball et al., 1994; Ho, 2001; Ormerod \& Ridgway, 1999) showed that novices tended to stick to a specific solution until they failed to handle it while experienced engineers tended to make breadth-first exploration. 
The phrase of project realization involves "deciding among a set of alternatives and communicating elements of the final design through writing, sketching, creating instructions, and creating bills of materials" (Atman et al., 2007, p. 361). Radcliffe and Lee (1989) showed the effectiveness of project realization influenced the quality of the design. Although there is no study comparing experts with novices in project realization, Atman and colleagues (2007) conducted a study comparing engineering freshmen with engineering seniors in engineering design and they found that senior students spent more time on project realization than freshmen.

\section{Protocol Analysis}

Protocol analysis is a methodology for capturing subjects' thoughts by eliciting their verbal reports (Williams et al., 2011). It is extensively applied to help researchers understand the designer's cognitive behavior, and is widely used in investigating the design process (Chrysikou \& Weisberg, 2005; Gericke, Schmidt-Kretschmer, \& Blessing, 2007; Goldschmidt, 2003; Kavakli \& Gero, 2002; Kolfschoten \& de Vreede, 2009; McDonnell \& Lloyd, 2007), especially the engineering design process (Ball, Ormerod, \& Morley, 2004; Cardella, Atman, Turns, \& Adams, 2008; Christensen \& Schunn, 2007;

Cross, 2002; Dorst \& Cross, 2001). 
There are two types of protocol analysis: think aloud and retrospective (Ericsson \& Simon, 1993). Subjects are required to speak out about anything they are thinking when performing the task in think aloud protocol. In retrospective protocol, subjects verbalize what they think after the task while viewing the video of performing. When there are two or more subjects participating in an activity, subjects express their thinking through natural conversation. In this situation, researchers use their conversation as verbal protocol data instead of using retrospective method.

Thinking aloud protocol is also called Verbal Protocol Analysis (VPA). Researchers started to use it in psychological research in the 1920s for examining problem solving. With the development of technology, audio and video became part of VPA to provide more accurate information. Ericsson and Simon's text (Ericsson \& Simon, 1993) guides most modern studies using VPA. They studied the validity of this research method and concluded that VPA could collect data accurately without affecting the performance of the subjects observed. They also believed that thinking aloud might slow down the working process but subjects' thinking was not interfered by thinking aloud unless they were asked to provide more information.

Some researchers cast doubt on the trustworthiness of VPA (Leighton, 2004). The major criticism is about the completeness of the data. VPA assumes that subjects report 
short-term memory instead of mental processes (Green, 1998), hence subjects cannot report information that is not available to short-term memory (Crisp, 2008). VPA also assumes that people have the ability to speak whatever they think, but this assumption has not been proven (Leighton, 2004). In addition, non-linguistic thoughts go faster than speaking, which means the speaking may slow down the thinking process (Crisp, 2008).

Ericsson and Simon also believed that the ability to vocalize varied from person to person (Ericsson \& Simon, 1993).

Nevertheless, protocol analysis provides considerable information compared with other methods. "Using the technique (protocol analysis), a researcher can obtain an insight into the subject's cognitive processes and use that to address a research question" (Hughes \& Parkes, 2003, p. 127). Alternative methods to study the design process are numerous, such as interviews and questionnaires. These methods may not provide as much information as VPA due to forgetfulness or selective reporting (Bainbridge, 1999; Ericsson \& Simon, 1993). In studies about individuals, there is usually a facilitator who reminds the individual to speak out whatever he or she thinks when performing the task. When there are multiple participants performing the task, the process of verbalization naturally happens. 


\section{Summary}

The literature review provided a discussion of the teaching and researching of engineering design. An important issue: problem decomposition/recomposition was brought up in the context of engineering design and the issue needs further investigated.

At the same time, problem decomposition/recomposition should be studied in the context of collaborative engineering design because it is a similar environment to practical engineering design where participants work in dyads as they complete the design challenge.

Experts vs. Novice and protocol analysis are commonly used techniques in studying expertise which provide the basic methodology of further research while FBS framework is a tool to quantitative measure the design process. The successful use of above techniques and tools facilitates building a solid methodology to investigate the issue discussed, which helps researchers have better understandings of design thinking from a design cognition perspective. It can contribute to the improvement of engineering design curriculum. 


\section{CHAPTER III}

\section{METHODOLOGY}

This study investigated the engineering design process of problem

decomposition/recomposition. Although problem decomposition/recomposition had been

studied in previous research (Arvanitis et al., 2001; Ho, 2001), no research was found

focusing on problem decomposition/recomposition in the context of groups. Considering

that engineering education does not place enough importance on problem

decomposition/recomposition (Ho, 2001) and engineers frequently work in groups in

industry, there is a need to study problem decomposition/recomposition in engineering

design.

This mixed-method research aimed at understanding the use of problem

decomposition/recomposition by engaging engineering freshmen, engineering seniors,

and engineering experts in an engineering design challenge. Dyads of college engineering

freshmen, dyads of college engineering seniors, and dyads of engineering experts

completed the same engineering design challenge and their design processes were

recorded through video and audio. After the completion of the design challenge, the

researcher conducted post-hoc focus group reflective interviews. Furthermore,

participants' sketches (artifacts) were collected to function as supplemental data. 
The verbal protocol data for each design dyad were documented, trancribed, segmented, and coded. In the coding section, a framework that combines FunctionBehavior-Structure (FBS) framework (Gero, 1990; Gero, Jiang, \& Williams, 2013; Lammi \& Becker, 2013) and the "levels of problem" was used. All data were analyzed using both quantitative and qualitative methods. The researcher quantitatively analyzed the data using descriptive techniques, including Mann-Whitney $\mathrm{U}$ tests and independent $t$ tests. In analyzing qualitative data, the researcher used the constant comparative method (Glaser, 1965) which includes four stages: “(1) comparing incidents applicable to each category, (2) integrating categories and their properties, (3) delimiting the theory, and (4) writing the theory" (Glaser, 1965, p. 439).

\section{Participants}

Fifty participants worked in 25 dyads $(N=25)$ took part in this study. All participants worked in dyads. There were 25 dyads in total. Among them, 20 were college engineering freshmen, 20 were engineering seniors, and 10 were engineering experts.

A literature review was conducted in order to choose an optimal group size. Large group sizes tend to have better diversity (Rau \& Heyl, 1990) but the conversations are usually dominated by two members of the group (Kan \& Gero, 2009). The researcher 
also considered whether to allow participants to form their own groups. Although the Moroney study (Moroney, Konz, \& Green, 2008) found that groups formed by students that know each other performed better than groups formed by instructors, Felder and Brent (2007) believed that instructors should form student groups instead because strong students tend to cluster and leave weak students. In addition, practicing engineers are not able to choose their teammates, so students need to learn to work with various group members (Felder \& Brent, 2007). Assigning participants into groups would benefit more students and can better approximate a real-world engineering design project. For this reason, student and expert participants in this study were assigned into groups.

\section{Participant Selection}

This study selected participants using purposeful sampling method (Gall, Gall, \& Borg, 2007), which can "select cases that are likely to be information-rich with respect to the purpose of the study" (p. 178). Based on the goal of the study and place of the study, all engineering freshmen and senior participants came from the College of Engineering at Utah State University. The College of Engineering had six departments and offered eight engineering bachelor degrees. During fall 2012, there were 1,718 undergraduate students and 438 graduate students studying in the College of Engineering. Students from the United States and other countries in the world attended this college. With the permission 
of engineering professors, the researcher solicited freshman engineering students from first-year engineering courses which included digital electronics, and computer engineering graphics. The senior engineering students were solicited from senior capstone engineering courses. With the consent of these instructors, the researcher sent introductory materials to the engineering students. Only engineering students could participate in this study, but there was no limitation on the engineering sub-discipline. Students voluntarily joined this study. Before participating, they signed an informed consent that is attached in Appendix F. Each student received a \$20 honorarium after their participation.

Expert engineer participants were selected from engineering companies in the Salt Lake City Area and Cache Valley Area of Utah as both were in close proximity to Utah State University. Engineering companies that participated in the study included Carollo Engineers, CH2M Hill, Icon Fitness, and Horrocks Engineers. Engineer participants had to have least 8 years engineering design experiences to participate in this study because the study needs engineers to be experts. Although the formation of expertise was much more than the accumulation of experiences and knowledge (Hoffman, 1996), Ericsson and Lehmann (1996) found that it typically took about 10 years or 10, 000 hours of practice to become an expert in a specific field. Assuming an engineer works 8 hours a 
day, 5 days a week, and 40 weeks a year, 10,000 hours equals to working for 6.25 years.

We took the average between 6 years and 10 years, and used 8 years as the standard to define an expert in this study. The researcher sent emails to potential expert engineer participants to introduce the purposes and procedures of the study and engineers voluntarily participated in the study. Before participating, they signed an informed consent which is attached in Appendix G. Each engineer received a \$50 honorarium after their participation.

\section{Institutional Review Board}

Before data collection, IRB approval was obtained from Utah State University and informed consent forms were received from individual participants. Student participants had to be 18 years or older. All participants were informed that they could withdraw from the research at any time during the study without repercussions. Student participants were further assured that no part of the research would effect their academic grades.

The researcher assigned each participant an ID number to represent his or her name and dyad. The data were associated with the ID numbers instead of participants' names. There was a coding sheet indicating the connection between ID number and participants' names. The researcher stored the coding sheet in a filing cabinet in the 
researcher's office accessible only to the researcher. The sheet would be destroyed permanently six months after data collection and analysis was completed.

\section{Data Collection}

\section{Overview}

All the student participants filled out an anonymous demographic questionnaire

prior to completing the engineering design challenge. The questionnaire included: gender, ethnicity, age, major, community description, the number of engineering courses taken, and GPA. Student participants completed their engineering design challenge in a laboratory at Utah State University. Using a laboratory reduced the amount of confounding variables such as unnecessary conversations and conversations with people outside design the dyad. Many studies involving verbal protocol analysis were conducted in the similar setting (Atman et al., 2007, 2008; Ho, 2001; Lammi, 2011; Mentzer \& Becker, 2010). The researcher examined and tested the audio and video devices before collecting data. They also scheduled an appropriate time that worked for the participants without affecting school. In order to ensure the reliability of the study, the researcher minimized the distractions. Participants did not have access to their phones, Facebook, emails or other media to communicate with other people during data collection. 
Engineering expert participants filled out an anonymous demographic

questionnaire before completing the design challenge. The questionnaire included: gender, ethnicity, age, major, and years of design experience. Considering the reliability of the study, expert participants completed their engineering design challenge in similar settings, such as a conference room in their company. The researcher scheduled an appropriate time that worked for participants without interfering with work time. Expert participants only communicated with their group member during data collection.

\section{Pilot study}

The pilot study in this research consisted of one dyad $(N=1)$ of students. The purpose was to test and optimize the process of the study. This included the design challenge, equipment, tools, and materials used in the study. The difficulty level of the design challenge, and the questionnaire and interview questions were also tested. The process of analyzing data was examined to evaluate the amount of codes generated by a dyad and how much time would be spent on coding data. The pilot resulted in modifications to the main study.

Two engineering freshmen students participated in the pilot study. The reason of choosing freshmen was to evaluate if the design challenge was within freshmen students' 
capabilities. The design challenge in this research had been used in other engineering design studies and most students should be familiar with the double hung windows. There were concerns if students could come up with a sufficient design within the limitation of time. Students in the pilot study were not very familiar with the double hung window but could understand the function and mechanics based on the instruction sheets and websites provided. Their understanding showed through their sketches, discussions, and their final design proposal. The researcher further confirmed it through the interview after the design challenge. Students in the pilot study submitted their final design proposal in 63 minutes, which was 3 minutes more than recommended time. The design had a sufficient quality and students went through the details of design requirements and completed their final product. Therefore, the one-hour limitation of the time was feasible. Equipment used in the pilot study consisted of two camcorders, an audio recorder, and a laptop. Students worked either in front of the white board or on the work surface of the table. There were two camcorders placed on professional tripods to captured students' movements from different angles. The audio recorder recorded students' conversations and was used as backup to the video recorders. The pilot study found that the audio recorded picked up students' conversations very clearly but the camcorders picked up background noises. In the stage of transcribing data, background noises could be 
compensated by the audio recorder but in order to make it easier to transcribe data, camcorders used in the pilot study were replaced with two camcorders of a higher quality. A 13-inch laptop was used in the pilot study to provide internet access. Students in the pilot study offered positive feedback on the laptop so it was sufficient for the purpose of the study. Pens, pencils, makers, an eraser, a ruler, a calculator, and some paper were provided to students to complete the design challenge. In the interview, students were satisfied with tools provided. The material provided was a design challenge sheet listing detailed information about the requirements of the design. Five websites provided by the design challenge were opened on the laptop so students could use them to find information they needed. The five websites included the construction of double-hung window, a Youtube video about the mechanism of double hung windows, a website about American Disabilities Act (ADA) information, a website of ADA Accessibility Guidelines for Buildings and Facilities (ADAAG), and a Wikipedia webpage about American with Disabilities Act of 1990. As mentioned earlier, students had limited access to prevent them from searching for solutions to the design problem. There were concerns about the information provided. The five websites might not provide all the information students needed, but exposing too much information can be difficult for students to manage. Based on students' discussion, sketches, and their final product, the 
five website provided were sufficient to assist students in completing the design. In the interview, students did express the need for information like the size and the weight of the window. Considering the purpose of the study was investigating participants' design thinking, an ill-structured design challenge would better serve the purpose. The researcher decided to keep the original design challenge and not to provide the dimension of the window to participants.

Students in the pilot study participated voluntarily. They received an email reminder the day before their participation. They did not have difficulties finding the conference room where data were collected. Students were asked to point out any unclear words or questions in both the questionnaire and interview. They indicated that they could understand all the questions clearly.

The dyad in the pilot study generated 537 utterances. Previous studies (Gero \& Kan, 2009; Kan \& Gero, 2010) obtained at least two hundred code segments for each participant unit. So it was concluded that the amount of codes generated from an hour's design activity was enough for the purpose of this research. The time spent on transcribing the data was seven to eight hours. As the transcribing process progresses, coders would be more proficient at transcribing and the amount of time spent would be less and less. This result was consistent with previous similar studies. 


\section{Design Challenge}

In this study, all dyads completed the same engineering design challenge that was open-ended and realistic. In the process of selecting the engineering design challenge for

the study, there were several issues considered. An easy-to-decompose challenge is ideal for this study, such as "a communication facility with answering function" used by Ho (2001) in investigating the use of problem decomposition/recomposition by individuals. But this type of challenges usually requires the knowledge in a specific field. For example, the challenge used by Ho is a complex electrical engineering design challenge and the novice in his study was a Masters level student in electrical engineering. In this study, however, student participants included both senior and freshmen. Most of the freshman students did not have the knowledge required to complete a complex design challenge. In addition, compared with a decomposable design challenge, a challenge that is not easy to decompose allowed designers to use various methods to decompose and recompose the problem, which would provide richer data for this study. 
The engineering design challenge used in this study was a double-hung window opener that assisted the elderly with raising and lowering windows. This challenge had been used by Gero (2010b) and other researchers to study engineering design. There were various engineering and social constraints in this challenge, which made it a typical engineering design challenge. In addition, double-hung windows were commonly used and most students were familiar with window operation and function so they did not need advanced engineering knowledge or background to complete the design challenge.

Participants were given a time period of one hour to complete the engineering design challenge. Instead of presenting practical products by the end of design, participants only submitted design proposals as their final outcomes. There was no instruction about the form or the content of the proposals they would submit. They did not build, test, and analyze their design because of the time constraint. See Appendix B for the engineering design challenge that was used in this study.

\section{Data Collection Process}

Before data collection began, the researcher entered the room where the engineering design challenge was to take place and set up audio and video devices for the data collection. They prepared a table, which was the major working space, for participants. The table was large enough for both members in the dyad to work together. 
The researcher placed all the tools needed on the table, including markers, pencils, calculators, and note pads. Two copies of the design challenge were placed on the table as well. The table was placed near a white board so participants could sketch ideas as they worked through their design. The researcher prepared two camcorders. They were placed where they could record participants' verbal protocol and actions from different angles, as well as participants' sketches on the white board. There was a computer in the room with five websites related to the design challenge opened. Participants could search for information on those websites but they were asked not to open other websites to search for information. The reason for having limited access to the internet was to make sure that participants would not use it to find a solution from the internet then apply it directly to the design challenge. The researcher tested all the devices before data collection began.

The primary form of data collection was protocol analysis. In the process of engineering design, the conversation naturally happened. The researcher recorded participants' conversations by audio and nonverbal interactions by video without answering participants' questions. The audio and video data complemented each other to provide rich information about the conversations and actions in engineering design 
process. Participants' sketches created during engineering design challenge supplemented the protocol data.

Immediately after completing the design challenge, participants took part in a focus group interview in which they answered questions from the researcher. Questions consisted of how participants framed the problem, generated alternative solutions, reached agreements, and used strategies. See Appendix C for the questions of the interview. The three types of data including verbal protocol data, sketching data, and interview data were triangulated to answer research questions of the study.

\section{Data Analysis}

After participants finished their design challenges, audio data were transcribed, segmented, and coded. In order to ensure the inter-coder reliability, two coders were involved in coding data. One of the coders was a graduate student and the other was an undergraduate student from Utah State University. The coders were trained using sample data from previous studies before coding data. The inter-coder reliability was calculated among coders to examine the reliability of coding.

Data Transcribing and Segmenting. Video and audio data were manually transcribed into spread sheets. The spread sheet data which contained designers' conversations and motions, were further broken into utterances. An utterance refereed to 
a unit of sentences that were spoken by a participant. Utterances were identified by a certain period of silence or the change of speakers.

After the process of transcribing, the utterances were segmented. Many available bases could be used but in this study, the segmentation was based on design issues. Each segment can only contain one code. For example, if multiple sentences focused on the same design issue, those sentences were segmented as one segment. If a sentence contained multiple design issues, the sentence was segmented as multiple segments.

Coding. The coding system had two dimensions: FBS ontology and "levels of the problem." The FBS ontology represented the process of design and was developed by Gero and colleagues (Gero, 1990; Gero and Kannengiesser, 2004). As illustrated in the literature review, Function (F) represented designers' expectations of the products, Behavior (B) represented the ways designers accomplish their goals, and Structure (S) represented the solutions to the problem. Under the first dimension, there was the second dimension of the coding system to represent the level of the problem. Engineers decomposed the design problem into multiple sub-problems and work on each subproblem in order to find out a solution. The level of the problem ranged from 1 to 3 . The meanings of each number are shown in Table 1. 
Table 1

Level of the Problem

\begin{tabular}{ll}
\hline Level of the Problem & Definition \\
\hline 1: System & Designers consider the problem as an integral whole. \\
$\begin{array}{ll}\text { 2: System and subsystems } & \text { Designers consider interactions between subsystems. } \\
\text { 3: Subsystems } & \text { Designers consider details of the subsystems. }\end{array}$ \\
\hline
\end{tabular}

Ho (2001) used a similar coding system to investigate engineering design

strategies used by individual electrical engineers. Gero and Mc Neill (1998) adopted the

coding system in analyzing design protocols. There is no data available for this study, but Ho’s study (2001) collected very similar data.

For each dyad of data in this research, each coder coded $65 \%$ of data with $30 \%$

overlap. The first $10 \%$ at the beginning were coded by both coders. The next $35 \%$ of data were coded by the first coder. The $10 \%$ data in the middle were coded by both coders.

The next $35 \%$ of data were coded by the second coder. The $10 \%$ at the end were coded by both coders. The coders performed the entire coding process in Microsoft Excel 2007. Each segment only had one code. The agreement between coders stayed above $80 \%$ after the training session. The overall agreement between coders expected was $80-85 \%$. 
The process of coding data included two coding system: FBS framework and

"levels of the problem." FBS framework had following codes: function (F), expected

behavior (Be), behavior from the structure (Bs), structure (S), documentation (D), and

requirement $(\mathrm{R})$. Their definitions were provided in this chapter. For those utterances that

did not fit into any definition of above codes, coders coded them as "other" $(\mathrm{O})$. Table 2

shows a piece of data coded by two coders. The last column shows arbitrated codes,

which are also the final codes of FBS ontology.

Table 2

Example of FBS Codes and Arbitrated Codes

\begin{tabular}{|c|c|c|c|c|}
\hline Subject & Utterance & $\begin{array}{l}\text { FBS } \\
\text { Coder } 1\end{array}$ & $\begin{array}{l}\text { FBS } \\
\text { Coder } 2\end{array}$ & $\begin{array}{l}\text { FBS Final } \\
\text { Code }\end{array}$ \\
\hline A & Not what I'm asking, but like how in-depth? & $\mathrm{F}$ & $\mathrm{R}$ & $\mathrm{F}$ \\
\hline A & $\begin{array}{l}\text { Because that's like how I'm in senior } \\
\text { drawing ... }\end{array}$ & $\mathrm{O}$ & $\mathrm{O}$ & $\mathrm{O}$ \\
\hline A & $\begin{array}{l}\text { Like a pulley is just something you go to the } \\
\text { store and buy. Like you... You know...Based } \\
\text { on, like }\end{array}$ & $\mathrm{R}$ & $S$ & $\mathrm{R}$ \\
\hline A & $\begin{array}{l}\text { I don't we are given all the numbers that we } \\
\text { need to be able to figure what type of pulley } \\
\text { system or what gear ratio. }\end{array}$ & $\mathrm{S}$ & S & S \\
\hline B & Yes, and the cost of materials & $\mathrm{S}$ & $S$ & $\mathrm{~S}$ \\
\hline A & $\begin{array}{l}\text { Yes, I mean, I wondering this is more just } \\
\text { given what we are given. I don't think we } \\
\text { really can say we need a specific number }\end{array}$ & $\mathrm{R}$ & $\mathrm{R}$ & $\mathrm{R}$ \\
\hline B & $\begin{array}{l}\text { I see, like } 15 \text { or whatever the number type } \\
\text { going here }\end{array}$ & S & S & S \\
\hline
\end{tabular}


For "levels of the problem," there were three numbers that represented the problem level designers worked on. The coding of this system was based on codes of FBS ontology. After coders finished coding using FBS ontology, they further coded Function $(\mathrm{F})$, expected Behavior $(\mathrm{Be})$, behavior from the structure (Bs), and Structure (S) using "levels of the problem." There are 6 codes in the FBS ontology in total. The reason $\mathrm{D}, \mathrm{R}$, and $\mathrm{O}$ were excluded from being coded with the level of the problem was the level of the problem reflected designer's cognitive activities. Based on their definitions, Code $\mathrm{D}, \mathrm{R}$, and $\mathrm{O}$ do not pertain to levels of the problem. Table 3 shows a piece of data coded by two coders. The coding of "levels of the problem" was based on codes of FBS ontology. The last column shows arbitrated codes, which are also the final codes of "levels of the problem." The process of arbitrating is further explained in next chapter. 
Table 3

Sample of Codes for Levels of the Problem

\begin{tabular}{|c|c|c|c|c|c|}
\hline Subject & Utterance & $\begin{array}{l}\text { FBS } \\
\text { Final } \\
\text { Code }\end{array}$ & $\begin{array}{l}\text { Levels } \\
\text { of the } \\
\text { Problem } \\
\text { Coder } 1\end{array}$ & $\begin{array}{l}\text { Levels of } \\
\text { the } \\
\text { Problem } \\
\text { Coder } 2\end{array}$ & $\begin{array}{l}\text { Levels of } \\
\text { the Problem } \\
\text { Final Code }\end{array}$ \\
\hline A & $\begin{array}{l}\text { Not what I'm asking, but like how in- } \\
\text { depth? }\end{array}$ & $\mathrm{F}$ & 1 & 1 & 1 \\
\hline A & $\begin{array}{l}\text { Because that's like how I'm in senior } \\
\text { drawing ... }\end{array}$ & $\mathrm{O}$ & - & - & - \\
\hline A & $\begin{array}{l}\text { Like a pulley is just something you go to } \\
\text { the store and buy. Like you.. You } \\
\text { know...Based on, like }\end{array}$ & $\mathrm{R}$ & - & - & - \\
\hline A & $\begin{array}{l}\text { I don't we are given all the numbers that } \\
\text { we need to be able to figure what type of } \\
\text { pulley system or what gear ratio. }\end{array}$ & $S$ & 3 & 3 & 3 \\
\hline B & Yes, and the cost of materials & $\mathrm{S}$ & 3 & 1 & 1 \\
\hline A & $\begin{array}{l}\text { Yes, I mean, I wondering this is more } \\
\text { just given what we are given. I don't } \\
\text { think we really can say we need a } \\
\text { specific number }\end{array}$ & $\mathrm{R}$ & - & - & - \\
\hline B & $\begin{array}{l}\text { I see, like } 15 \text { or whatever the number } \\
\text { type going here }\end{array}$ & $\mathrm{R}$ & - & - & - \\
\hline
\end{tabular}

The numbers of utterances generated by each dyad were different, so simply

comparing the frequencies of each type of code would affect the validity of the study.

The percentages of codes were used in order to compare the differences among dyads.

The percentage of each code from each dyad was calculated by dividing the frequency of the code into the total number of effective codes of the dyad. For the FBS ontology, the total number of effective codes was calculated by subtracting the number of code $\mathrm{O}$ from the total number of codes. See Table 4 for the calculation of percentages of each code in 
FBS ontology. For "levels of the problem," the total number of effective codes was

calculated by subtracting the numbers available of code $\mathrm{O}, \mathrm{R}$, and $\mathrm{D}$ from the total

number of codes. See Table 5 for the calculation of percentages of each code in "levels of

the problem."

Table 4

Calculation of Percentages of Codes for FBS Ontology

\begin{tabular}{ll}
\hline Name & Calculation \\
\hline $\begin{array}{l}\text { Effective Utterances for } \\
\text { FBS Ontology (EFBS) }\end{array}$ & EFBS $=$ TL $-\mathrm{NO}$ \\
Percentage of F (PF) & $\mathrm{PF}=\mathrm{NF} / \mathrm{EFBS}$ \\
Percentage of Be (PBe) & $\mathrm{PBe}=\mathrm{NBe} / \mathrm{EFBS}$ \\
Percentage of Bs $(\mathrm{PBs})$ & $\mathrm{PBs}=\mathrm{NB} / \mathrm{EFBS}$ \\
Percentage of S (PS) & $\mathrm{PS}=\mathrm{NS} / \mathrm{EFBS}$ \\
Percentage of D (PD) & $\mathrm{PD}=\mathrm{ND} / \mathrm{EFBS}$ \\
Percentage of R (PR) & $\mathrm{PR}=\mathrm{NR} / \mathrm{EFBS}$ \\
\hline
\end{tabular}

*Available data included: Number of F (NF), Number of Be (NBe), Number of Bs (NBs), Number of S (NS), Number of D (ND), Number of R (NR), Number of O (NO), and Total Number of Utterances (TL). 
Table 5

Calculation of Percentages of Codes for Levels of the Problem

\begin{tabular}{ll}
\hline Name & Calculation \\
\hline Effective Utterances for Levels of the & EL $=$ N1 + N2 + N3 \\
Problem (EL) & Or EL = TL $-\mathrm{NO}-\mathrm{NR}-\mathrm{ND}$ \\
& Or EL $=\mathrm{NF}+\mathrm{NBe}+\mathrm{NBs}+\mathrm{NS}$ \\
Percentage of 1 (P1) & $\mathrm{P} 1=\mathrm{N} 1 / \mathrm{EL}$ \\
Percentage of 2 (P2) & $\mathrm{P} 2=\mathrm{N} 2 / \mathrm{EL}$ \\
Percentage of 3 (P3) & $\mathrm{P} 3=\mathrm{N} 3 / \mathrm{EL}$ \\
Percentage of Decomposition (PDe) & $\mathrm{PDe}=\mathrm{NDe} / \mathrm{EL}$ \\
Percentage of Recomposition (PRe) & $\mathrm{PRe}=\mathrm{NRe} / \mathrm{EL}$ \\
\hline
\end{tabular}

*Available data included: Total Number of Utterances (TL), Number of 1 (N1), Number of 2 (N2), Number of 3 (N3), Number of Decomposition (NDe), Number of Recomposition (NRe), Number of R (NR), and Number of O (NO).

Quantitative Data. Overview. The process of coding was quantifying verbal protocol data from the design challenge. The researcher used statistical methods to analyze coded data. The researcher applied tests in analyzing quantitative data according to the quality of the data (Corder \& Foreman, 2009; Drew, Hardman, \& Hosp, 2008). There were three types of data: data from engineering freshmen dyads, data from engineering seniors, and data from engineering expert dyads.

1. Research question 1: Do engineering experts use problem decomposition and recomposition more than engineering seniors and engineering freshmen in engineering design? 
To answer Research Question 1, the process of problem decomposition and problem recomposition needed to be identified. Table 6 shows a piece of sample data of an individual dyad. The level of the problem designers worked on transitioned among different levels through the time. As previously illustrated, the problem decomposition is a top-down process while the problem recomposition is a bottom-up process. When the level of the problem transitions from a higher level to a lower level, it is defined as the problem decomposition, and when it transitions from a lower level to a higher level, it is defined as the problem recomposition. Table 6 provided a piece of data where problem decomposition and problem recomposition were coded.

Using above process, percentages of frequencies of problem decomposition and recomposition of each dyad were calculated, and then means and standard deviations were calculated across three types of dyads. In order to tell if there were statistically significant differences among three dyads of data, the researcher compared them using statistical tests. The results answered Research Question 1. 
Table 6

\begin{tabular}{|c|c|c|c|c|}
\hline Subject & Utterance & $\begin{array}{l}\text { FBS Final } \\
\text { Code }\end{array}$ & $\begin{array}{l}\text { Levels of the } \\
\text { Problem } \\
\text { Final Code }\end{array}$ & $\begin{array}{l}\text { Decomposition/ } \\
\text { Recomposition }\end{array}$ \\
\hline A & $\begin{array}{l}\text { Not what I'm asking, but like how in- } \\
\text { depth? }\end{array}$ & $\bar{F}$ & 1 & - \\
\hline A & $\begin{array}{l}\text { Because that's like how I'm in senior } \\
\text { drawing ... }\end{array}$ & $\mathrm{O}$ & - & - \\
\hline A & $\begin{array}{l}\text { Like a pulley is just something you go to } \\
\text { the store and buy. Like you.. You } \\
\text { know...Based on, like }\end{array}$ & $\mathrm{R}$ & - & - \\
\hline A & $\begin{array}{l}\text { I don't we are given all the numbers that } \\
\text { we need to be able to figure what type of } \\
\text { pulley system or what gear ratio. }\end{array}$ & $S$ & 3 & $\mathrm{D}$ \\
\hline B & Yes, and the cost of materials & $S$ & 1 & $\mathrm{R}$ \\
\hline A & $\begin{array}{l}\text { Yes, I mean, I wondering this is more } \\
\text { just given what we are given. I don't } \\
\text { think we really can say we need a } \\
\text { specific number }\end{array}$ & $\mathrm{R}$ & - & - \\
\hline B & $\begin{array}{l}\text { I see, like } 15 \text { or whatever the number } \\
\text { type going here }\end{array}$ & $\mathrm{R}$ & - & - \\
\hline
\end{tabular}

2. Research question 2: Which level of the problem do engineering experts, seniors, and freshmen spend most cognitive effort on?

The researcher first calculated the percentages of coding on each level of the problem of each dyad, then means and standard deviations across the three types of dyads were calculated. In order to answer the question, the researcher compared their means using statistical tests to calculate if there were statistically significant differences among the three types of dyads. 
3. Research question 3: What mental activities are used by engineering experts, seniors, and freshmen when they approach different levels of the problem?

To answer this question, the researcher first calculated the percentages of codes in FBS ontology on each level of the problem within each dyad. After obtaining data from each dyad, the researcher calculated means and standard deviations across three types of dyads. The researcher ran statistical tests to calculate if there were statistically significant differences among three types of dyads.

Qualitative Data. As stated by Lichtman, In general, the main purpose of qualitative research — whatever kind —is to provide an in-depth description and understanding of the human experiences. It is about humans. Qualitative research has as its purpose a description and understanding of human phenomena, human interaction, or human discourse. (Lichtman, 2006, p. 8)

The analysis of qualitative data was connected with quantitative data. In this mixed method study, the qualitative data included: sketches from the design challenge and the interview data. These data were triangulated against each other. The analysis of qualitative data used the constant comparative method (Glaser, 1965). This method is not limited to use in analyzing qualitative data. As Glaser described, there are four stages of 
the constant comparative method: "(1) comparing incidents applicable to each category, (2) integrating categories and their properties, (3) delimiting the theory, and (4) writing the theory" (Glaser, 1965, p. 439).

This study followed above steps in analyzing qualitative data. The first step included going through each interview and sketch. As the researcher went through interview and sketch data, themes were recorded. The second step was sorting themes to different categories. The third step was looking for differences among expert dyads, senior dyads, and freshman dyads within each category. The last step was writing up findings. The analysis of qualitative data allowed any themes or new phenomena to emerge that could not be discovered by analyzing quantitative data.

Table 7 provided a small piece of interview data in order to show the first three steps of constant comparative method used in analyzing qualitative data. There were much more themes and categories emerged from the entire qualitative data and some themes emerged repeatedly. The conclusions drawn in Step 3 were based on the analysis of the entire data instead of the small piece shown in Table 7. The fourth step of analyzing qualitative data was writing the theory which is not shown in Table 7 . The analysis of sketches followed the same four steps. 
Table 7

\section{Example of Qualitative Data Analysis}

\begin{tabular}{|c|c|c|c|}
\hline $\begin{array}{l}\text { Interview } \\
\text { Question }\end{array}$ & $\begin{array}{l}\text { Step 1: Themes Emerged from Answers F- } \\
\text { Freshmen S - Seniors E - Engineers }\end{array}$ & Step 2: Categorizing & $\begin{array}{l}\text { Step 3: Comparing } \\
\text { students and engineers }\end{array}$ \\
\hline \multirow{7}{*}{$\begin{array}{l}\text { How did you } \\
\text { define the } \\
\text { problem? }\end{array}$} & Increase the force $(\mathrm{F})$ & Problem definition & \multirow{14}{*}{$\begin{array}{l}\text { Problem definition: } \\
\text { Engineers understood } \\
\text { the problem better than } \\
\text { students. } \\
\text { Design Experiences: } \\
\text { Engineers had more } \\
\text { design experiences and } \\
\text { tent to use their } \\
\text { experience in solving } \\
\text { new problems. }\end{array}$} \\
\hline & Modify existing window $(\mathrm{F})$ & Problem definition & \\
\hline & ADA Guidelines (S) & Problem definition & \\
\hline & Old people in wheel chairs (S) & Problem definition & \\
\hline & $\mathrm{ADA}$ requirements $(\mathrm{E})$ & Problem definition & \\
\hline & No major construction required (E) & Problem definition & \\
\hline & Safety issues (E) & Problem definition & \\
\hline \multirow{5}{*}{$\begin{array}{l}\text { How did you } \\
\text { know which } \\
\text { ideas would } \\
\text { work and } \\
\text { which would } \\
\text { not work? }\end{array}$} & Daily experiences $(\mathrm{F})$ & Design experiences & \\
\hline & $\begin{array}{l}\text { Don't know if the ideas would work and } \\
\text { how much it would cost }(\mathrm{F})\end{array}$ & Cost & \\
\hline & Analyze pros and cons $(\mathrm{S})$ & Alternative solutions & \\
\hline & $\begin{array}{l}\text { Comparing the design with devices used } \\
\text { before }(E)\end{array}$ & Design experiences & \\
\hline & Analyze clients (E) & Problem definition & \\
\hline \multirow{4}{*}{$\begin{array}{l}\text { Why and how } \\
\text { did you } \\
\text { choose your } \\
\text { final idea or } \\
\text { plan? }\end{array}$} & Practical and feasible $(\mathrm{F})$ & Alternative solutions & \\
\hline & Easy to work $(\mathrm{F})$ & Alternative solutions & \\
\hline & $\begin{array}{l}\text { Consider how solutions fit requirements } \\
\text { (S) } \\
\text { Only came up with one idea (S) }\end{array}$ & Alternative solutions & \multirow{9}{*}{$\begin{array}{l}\text { Cost: Students did not } \\
\text { pay enough attention to } \\
\text { the cost of the design. } \\
\text { Some of them did not } \\
\text { know the cost of } \\
\text { materials. } \\
\text { Alternative solutions: } \\
\text { Engineers evaluated } \\
\text { alternative solution } \\
\text { more effective than } \\
\text { students. }\end{array}$} \\
\hline & $\begin{array}{l}\text { Cost effective (S) } \\
\text { Easy to use and maintenance (E) } \\
\text { Fits the goal of the design (E) } \\
\text { Not block views (E) }\end{array}$ & $\begin{array}{l}\text { Cost/ Problem definition } \\
\text { Problem definition } \\
\text { Alternative solutions } \\
\text { Problem definition }\end{array}$ & \\
\hline \multirow{7}{*}{$\begin{array}{l}\text { What } \\
\text { difficulties } \\
\text { did you meet } \\
\text { in solving the } \\
\text { problem? }\end{array}$} & $\begin{array}{l}\text { Have difficulties deciding which solution } \\
\text { to choose }(\mathrm{F})\end{array}$ & Alternative solutions & \\
\hline & $\begin{array}{l}\text { They are good at solving homework } \\
\text { problems but not the real problems. (F) }\end{array}$ & $\begin{array}{l}\text { Homework problem and } \\
\text { real life problems }\end{array}$ & \\
\hline & $\begin{array}{l}\text { The design challenge is very different from } \\
\text { problems in class. }(F)\end{array}$ & $\begin{array}{l}\text { Homework problem and } \\
\text { real life problems }\end{array}$ & \\
\hline & Need more constraints and criteria $(\mathrm{S})$ & Problem definition & \\
\hline & They don't know the cost of materials (S) & Cost & \\
\hline & $\begin{array}{l}\text { They need more information about clients' } \\
\text { design consideration (E) }\end{array}$ & Problem definition & \\
\hline & $\begin{array}{l}\text { They wanted to talk to a window producer } \\
\text { to make a product of their design (E) }\end{array}$ & Design experiences & \\
\hline
\end{tabular}




\section{Summary}

This study applied a mixed methodology to answer research questions. The verbal

protocol data from engineering design challenge, participants' sketches from the design, and focus group interview data were triangulated with each other to provide rich information about engineering design and how participants used problem decomposition/recomposition in the design process. The verbal protocol data from engineering design challenge was transcribed, segmented and coded using developed FBS ontology and "levels of the problem." The results of the study will be shared with other researchers in this field through publications.

The study quantitatively characterized the design thinking of both engineering students and expert engineers from a design cognition perspective and measure the differences between the design cognition of engineering students and expert engineers. It adds to the knowledge of the development of design thinking across engineering education and experience, and proposes educational interventions that narrow the differences between novices and experts. 


\section{CHAPTER IV}

\section{RESULTS}

This research focused on understanding differences of using problem

decomposition and problem recomposition among engineering freshmen, engineering

seniors, and engineering experts. Audio and video data were collected. Video data, audio

data, and participants' movements were transcribed, segmented, and coded using FBS

framework and "levels of the problem." These data are qualitative but the process of

coding quantified data so they worked as quantitative data in this study. Data from

interviews and design sketches functioned as qualitative data. The results of both

quantitative and qualitative data helped to answer the research questions of the study.

This chapter will present and discuss the results of the study.

\section{Participants}

Fifty participants worked in dyads $(N=25)$ participated in this study. Eight of

them were female and 42 of them were males. Forty-seven of them identified their race as

"White," two of them identified their race as "Hispanic," and one of them identified his

race as "other." Participants' mean $(\mathrm{M})$ age was 25.38 years with a standard deviation of

6.96. The youngest participant was 18 years old and the oldest participant was 53. See 
Appendix D for the Anonymous Demographic Questionnaire for students and Appendix

E for Anonymous Demographic Questionnaire for engineers.

There were 20 engineering students who participated in the study. They were placed in dyads and classified as freshmen. They were all students from College of Engineering in Utah State University. Eleven of them were freshmen and 9 of them were first-semester sophomore. None of them had taken any advanced engineering courses when participating in this study. Their mean $(M)$ age was 21.60 years with a standard deviation of 2.74. Their mean $(M)$ GPA was 3.56 with a standard deviation of .39 .

Another 20 engineering students participated in the study and were placed in dyads and classified as seniors. They were all students from College of Engineering in Utah State University. They were all senior students and had taken engineering design courses by the time they participated in the study. Their mean $(M)$ age was 23.85 years with a standard deviation of 1.42 . Their mean $(M)$ GPA was 3.59 with a standard deviation of .32 .

There were 10 professional engineers that participated in this research. Four of them were from Icon Fitness, two of them were from $\mathrm{CH} 2 \mathrm{M}$ Hill, two of them were from EL Dorado Engineering, and two of them were from Horrocks Engineers. Their mean $(M)$ 
age was 38.63 years with a standard deviation of 7.17. Their mean $(M)$ years of engineering design was 13.62 years with a standard deviation of 7.61.

\section{Coder Training}

Two coders were used to transcribe, segment, and code data. The researcher worked as one of the coders. Coders were trained to code FBS framework first. The researcher received FBS training from Dr. John Gero who developed the FBS framework before the data were collected. The training was performed at George Mason University in July, 2012. Four researchers from different institutions took part in the training. Dr.

Gero started the training by introducing the FBS ontology. He also stated the definition of each code and provided a few examples of codes. After that, he introduced the entire process of using FBS ontology to analyze design behaviors, including how to transcribe, segment, code, and arbitrate data. He gave instruction on how to use LINKORDER, which is a program widely used in analyzing FBS data.

In the training, Dr. John Gero spent a majority of time teaching coding techniques.

He first showed a piece of video data that was from his previous research. In the video, two students were working on the double-hung window opener design challenge. Dr. John Gero provided an excel file on which the video data were transcribed and segmented into spread sheets. He asked researcher trainees to start coding the first 10 segments. 
There were many disagreements among trainees. Dr. Gero went through each segment and asked trainees to state their codes and their reasons of coding. As trainees stated their thoughts, Dr. Gero expressed his opinions as an experienced researcher. Trainees continued to code another 10 codes. After that, Dr. Gero repeated the previous process of arbitrating. The number of disagreements was less than the first round. Due to the limitation of time, the training process finished before an ideal inter-coder reliability (80\%) was reached. According to Dr. Gero, who had trained a number of coders to use the FBS ontology, a higher inter-coder reliability would be reached by repeating the arbitrating process.

In this study, training materials and sample data were used from the previous training. The researcher performed the training and participated in the arbitrating process because she was one of the coders. The researcher started training by introducing the overview of FBS framework to the undergraduate coder. After that, the researcher went through several examples of each code.

When the undergraduate coder was familiar with the FBS ontology, the arbitrating process started. For each segment of sample data, both coders coded and provided reasons why they chose the code. When there was a disagreement, they looked up 
references and negotiated until they reached an agreement. They followed this pattern and coded 30 segments of the sample data.

In the next section of training, every continuous 10 segments composed a group, and each coder coded a group separately. Coders compared their codes and when there were disagreements, they went through the negotiation process until reaching agreements. Coders repeated the process for 20 groups. For the first several groups, the inter-coder reliability were not very high $(50-70 \%)$. As coders continued coding, they shared more understandings about the framework and definitions of codes. By the $14^{\text {th }}$ group, the inter-coder reliability remained above $70 \%$, which is acceptable in social science (Schloss \& Smith, 1999).

In the next section of training, each of coder coded 50 segments separately. After coding, they compared their codes and calculated the inter-coder reliability, which was $82 \%$. They repeated the process with another 50 segments and the inter-coder reliability was $84 \%$. At this point, they had reached the accepted inter-coder reliability in social science, which was higher than $70 \%$ (Schloss \& Smith, 1999). So they were ready to use FBS framework to code data of the study.

After the FBS training, coders were trained to use a coding system of "levels of the problem." As mentioned earlier, "levels of the problem" only applied to segments 
coded as F, Be, Bs, and S. Neither of coders had received prior training to use this system and there were no training materials specifically designed for this coding system. For this reason, the researcher followed steps in FBS training to reach a high inter-coder reliability in coding "levels of the problem."

The training of using "levels of the problem" started by having two coders read literature about this coding system. They started the arbitrating process by coding every single segment of the sample data. Both coders coded sample data and provided reasons of why their chose one code over others. When there was a disagreement, they went through the arbitration process until an agreement was reached. They followed this pattern and coded the first 30 segments of the sample data.

In next section, every continuous 10 segments composed a group, and each coder coded a group separately. Coders compared their codes and when there were disagreements, they went through the negotiation process until reaching agreements. Coders repeated the process for 15 groups. For the first several groups, the inter-coder reliability was not very high. As coders continued coding, they shared more understandings about the framework and definitions of codes. By the $10^{\text {th }}$ group, the inter-coder reliability was $82 \%$. 
Next, each of coder coded 50 segments separately. After coding, they compared their codes to calculate the inter-coder reliability. The inter-coder reliability between two coders was $81 \%$. In order to further examine the reliability of coders, the consistency between the coder and arbitrated codes was calculated. The first coder's consistency between arbitrated codes was $82 \%$ and the second coder's consistency between arbitrated codes was $80 \%$.

In the next section, the coders repeated above process with another 50 segments. The inter-coder reliability between two coders was $86 \%$. The first coder's consistency between arbitrated codes was $89 \%$ and the second coder's consistency between arbitrated codes was $84 \%$. At this point, they had reached the inter-coder reliability required, which was higher than $70 \%$. They were now able to use "levels of the problem" to code data of the study.

\section{Coding Techniques}

For each dyad of data in this research, each coder coded $65 \%$ of data with $30 \%$ overlap. The first $10 \%$ at the beginning were coded by both coders. The next $35 \%$ of data were coded by the first coder. The $10 \%$ data in the middle were coded by both coders. The next $35 \%$ of data were coded by the second coder. The $10 \%$ at the end were coded by both coders. Participants' conversations were segmented according to design issues 
they discussed. During coding, multiple sentences were combined as a single segment, and a sentence could be divided into several segments.

For example, the following conversation is from two participants:

B: "I'm thinking a crowbar."

A: "Just give them all a crowbar?"

B: "Just give them a crowbar."

Three sentences were all about the issue of using a crowbar so they were

combined as a single segment. In the following sentence:

B: "Double pulley and then double pulley, that would be gobs of extra, but

I mean it just makes it nice..."

It contains two different design issues: double pulley and how double pulley work.

In this case, coders broke a sentence into two segments.

\section{Research Question 1}

The first research question was, "do engineering experts use problem

decomposition and recomposition more than engineering seniors and engineering freshmen in engineering design?" In order to answer this question, the video and audio data were segmented and coded from each dyad of participants. It was a process of 
quantifying qualitative data. Interview data and participants' sketches worked as

qualitative data to address this question.

Twenty-five dyads of data were collected, including 10 dyads of freshmen, 10

dyads of seniors, and 5 dyads of engineers. In total, 12527 segments were generated and

$10,546(84.19 \%)$ of them were coded within FBS framework. Those FBS codes were

further coded using "levels of the problem" and 8971 (85.06\%) of them were coded

within "levels of the problem." Using the method mentioned in Chapter III, the

percentages of using problem decomposition and recomposition of each dyad were

calculated. Means and standard deviations were calculated across the three types of dyads,

which are shown in Table 8.

Table 8

Means and Standard Deviations of Problem Decomposition and Problem Recomposition

\begin{tabular}{ccccc}
\hline Type of Dyad & \multicolumn{2}{c}{ Problem Decomposition (\%) } & \multicolumn{2}{c}{ Problem Recomposition (\%) } \\
\cline { 2 - 5 } & Mean & Standard Deviation & Mean & Standard Deviation \\
\hline Freshmen $(N=10)$ & 15.13 & 2.66 & 15.28 & 2.64 \\
Seniors $(N=10)$ & 15.22 & 3.27 & 15.31 & 3.14 \\
Engineers $(N=5)$ & 22.38 & 3.01 & 22.57 & 3.54 \\
\hline
\end{tabular}

To determine what statistical techniques to use, a normality test was run using

SPSS to see if the data were normally distributed. The histogram and probability plot 
showed that data of problem decomposition were slightly skewed. Data from senior dyads in problem decomposition were found not normally distributed since the $p$ value of Shapiro-Wilk test was smaller than 0.05. The $p$ values of Shapiro-Wilk test for other dyads of data were larger than 0.05 , which means these data were normality distributed. Based on this result, the researcher decided to use non parametric statistics when the comparison involves senior dyads in using problem decomposition. Since the data collected were independent, Mann-Whitney U test (confidence interval percentage $=95 \%$ ) was selected. When the comparison does not involve this dyad of data, independentsamples $t$ tests (confidence interval percentage $=95 \%$ ) were used. The results are shown in Table 9.

Table 9

Results of Research Question 1

\begin{tabular}{lcccc}
\hline $\begin{array}{l}\text { Types of Dyads } \\
\text { Compared }\end{array}$ & \multicolumn{2}{c}{ Problem Decomposition } & \multicolumn{2}{c}{ Problem Recomposition } \\
& $p$ Value & Effect Size & $p$ Value & Effect Size \\
\hline Freshmen vs. Seniors & .496 & N/A & .980 & N/A \\
Freshmen vs. Engineers & $.000^{* *}$ & 2.55 & $.001^{* *}$ & 2.33 \\
Seniors vs. Engineers & $.010^{* *}$ & 2.28 & $.001^{* *}$ & 2.17 \\
\hline
\end{tabular}

$* * b \leq 0.01$

In the use of problem decomposition, the mean of seniors is slight lower than

freshmen dyads, but there was no statistically significant difference, which means 
freshmen used problem decomposition as much as seniors did in engineering design. The mean of engineers in using problem decomposition is higher than both freshmen and seniors. The independent-samples $t$ tests showed that there is a statistically significant difference between engineers and freshmen, as well as between engineers and seniors. Therefore, engineers used more problem decomposition than both freshmen and seniors did in engineering design.

In the use of problem recomposition, the results are similar. The mean of seniors is slight lower than freshmen dyads, but the results of independent-samples $t$ tests showed there is no statistically significant difference, which means freshmen used problem recomposition as much as seniors did in engineering design. The mean of engineers in using problem recomposition is higher than both freshmen and seniors. The independentsamples $t$ tests showed that there is a statistically significant difference between engineers and freshmen, as well as between engineers and seniors. Therefore, engineers used more problem recomposition than both freshmen and seniors.

The analysis of qualitative data had similar findings. In the interview, a question was "Did you tackle the problem as a whole or decompose it into several sub-problems? If you decomposed it, why did you choose it over the other one?" Students' answers varied from dyad to dyad. Some dyads broke the problem into multiple sub-systems, 
some dyads solved the problem as a whole because they thought the problem was too simple to break, and some student dyads did both. For engineer dyads, the answers were more consistent. They started with considering the whole problem to get a big picture, and then they broke it into small pieces to work on, and finally they combined small pieces into the final solution.

Participants' sketches did not directly reflect how they used problem decomposition and problem recomposition in the design process. However, engineer dyads' sketches were found to be more balanced between the big picture and details. In engineers' sketches, they presented both the whole design idea and details of their design in the format of figures and words. The entire design idea was presented through stating their ideas in words, providing features and benefits of the design, figures of the window and the device they designed, the description of how the device worked in words and figures. The details of the design were presented through multiple views of the device, figures of parts, the sizes and numbers of each part, figures of connections of different parts, list of materials, and cost of materials. Freshman dyads' sketches lacked the presentation of the big picture. Generally speaking, their design ideas in words were rarely found, and figures describing the entire device were not clear. Compared with freshman dyads, senior dyads did a better job. Some of their sketches had words and 
figures describing the design ideas, but in general, the presentation of the entire idea was not as clear as engineer dyads'.

\section{Research Question 2}

The second research question was, “on which 'level of the problem' do engineering experts, seniors, and freshmen spend most cognitive effort?" In order to answer this question, the percentages of code on each level across dyads were calculated.

In the process of calculating percentages, the data from Dyad Engineers \#5 were found to be significantly different on Level 1 and 3. Because of this, the researcher ran SPSS to further determine if they were outliers. The following formula (1) and formula (2) were used to identify the boundaries of outliers:

$$
\begin{aligned}
& \text { Upper }=\mathrm{Q} 3+[2.2 *(\mathrm{Q} 3-\mathrm{Q} 1)] \\
& \text { Lower }=\mathrm{Q} 1-[2.2 *(\mathrm{Q} 3-\mathrm{Q} 1)]
\end{aligned}
$$

Q1 represents 25 percentile and Q3 represents 75 percentile. After identifying the boundaries of outliers, data from Dyad Engineers \#5 were not detected as outliers.

The raw data came from data coded by FBS framework. Part of the data was further coded using "levels of the problem." The reason was some codes in FBS framework were not relevant to "levels of the problem." FBS codes describe cognitive 
activities of designers. "Levels of the problem" was coded based on FBS codes so the distributions of "levels of the problem" reflect cognitive effort spent by designers.

There were 8971 segments coded within "levels of the problem." Among those codes, 1255 (13.99\%) of them were coded as Level 1, $1645(18.34 \%)$ of them were coded as Level 2, and $6071(67.67 \%)$ of them were coded as Level 3. Means and standard deviations were calculated across the three types of dyads, which are shown in Table 10.

Table 10

Means and Standard Deviations of "Levels of the Problem"

\begin{tabular}{lllllll}
\hline & \multicolumn{2}{l}{ Level 1 (\%) } & \multicolumn{2}{l}{ Level 2 (\%) } & \multicolumn{2}{c}{ Level 3 (\%) } \\
\cline { 2 - 7 } Type of Dyad & Mean & Standard Deviation & Mean & Standard Deviation & Mean & $\begin{array}{l}\text { Standard } \\
\text { Deviation }\end{array}$ \\
\cline { 2 - 7 } & 11.08 & 2.46 & 17.42 & 3.99 & 71.50 & 4.87 \\
\hline $\begin{array}{l}\text { Freshmen } \\
(\mathrm{N}=10)\end{array}$ & 12.59 & 3.57 & 16.75 & 4.61 & 70.66 & 4.37 \\
$\begin{array}{l}\text { Seniors } \\
(\mathrm{N}=10)\end{array}$ & 20.89 & 13.68 & 23.79 & 6.63 & 55.32 & 15.66 \\
$\begin{array}{l}\text { Engineers } \\
(\mathrm{N}=5)\end{array}$ & & & & & & \\
\hline
\end{tabular}

Mean and standard deviation from the three types of dyads show that freshmen, seniors, and engineers spent most of their cognitive effort on Level 3 (details of subsystems). All three types of dyads spent least cognitive effort on Level 1 (system). To further investigate if the amounts of cognitive effort spent on each level were different 
across the three types of dyads, statistical techniques were used to compare means among the dyads.

To determine what statistical techniques to use, a normality test was run using SPSS to see if the data were normally distributed. All dyads of data were found skewed to different degrees but not enough to influence the normality of the data. The $p$ value of Shapiro-Wilk test of above data were larger than 0.05 . Based on this result, the research decided to use independent-samples $t$ tests (confidence interval percentage $=95 \%$ ) to compare the means between different types of dyads. The results are shown in Table 11 . On Level 1, which indicates designer considering the problem as an integral whole, the mean of senior dyads is slight higher than freshman dyads, and the mean of engineers is much higher than freshmen and seniors. There were statistically significant differences between freshman dyads and engineer dyads, as well as senior dyads and engineer dyads. No significant difference was found between freshman dyads and senior dyads. The results show that freshman dyads and senior dyads spent same amount of cognitive effort when they considered the problem as an integral whole. Engineer dyads spent more cognitive effort than freshman dyads and senior dyads when they considered the problem as an integral whole. 
Table11

Results of Research Question 2

\begin{tabular}{lcccccc}
\hline Types of Dyads Compared & \multicolumn{2}{c}{ Level 1 } & \multicolumn{2}{c}{ Level 2 } & \multicolumn{2}{c}{ Level 3 } \\
\cline { 2 - 7 } & $p$ Value & $\begin{array}{c}\text { Effect } \\
\text { Size }\end{array}$ & $p$ Value & $\begin{array}{c}\text { Effect } \\
\text { Size }\end{array}$ & $p$ Value & $\begin{array}{c}\text { Effect } \\
\text { Size }\end{array}$ \\
\hline Freshmen vs. Seniors & .286 & N/A & .732 & N/A & .842 & N/A \\
Freshmen vs. Engineers & $.020^{*}$ & 1.00 & $.035^{*}$ & 1.16 & $.009^{* *}$ & 1.40 \\
Seniors vs. Engineers & $.043^{*}$ & 0.83 & $.031^{*}$ & 1.23 & $.009^{* *}$ & 1.33 \\
\hline
\end{tabular}

$* b \leq 0.05$

$* * b \leq 0.01$

On Level 2, which indicates designer considering interactions between

subsystems, the mean of freshmen is slight higher than seniors, but there was no

statistically significant difference found. This means freshmen spent their cognitive effort

as much as seniors did when they considered interactions between subsystems. The mean

of engineers on Level 2 is higher than both freshmen and seniors. The independent-

samples $t$ tests showed that there is a statistically significant difference between engineers

and freshmen, as well as between engineers and seniors. At the same time, the standard

deviation of engineering experts is higher than engineer freshmen's and engineer seniors',

which means the cognitive effort spent by engineer experts on Level 2 are spread out

over a large range of values. Therefore, on average, engineers spent more cognitive effort 
than freshmen and seniors did when they considered interactions between subsystems in engineering design.

On Level 3, which indicates designer considering details of subsystems, the mean of freshmen is slight higher than seniors, but there was no statistically significant difference found, which means freshmen spent their cognitive effort as much as seniors did when they considered details of subsystems. The mean of engineers on Level 3 is lower than both freshmen and seniors. The independent-samples $t$ tests showed that there is a statistically significant difference between engineers and freshmen, as well as between engineers and seniors. Therefore, engineers spent less cognitive effort than freshmen and seniors did when they considered details of subsystems in engineering design.

In the interview, one of the questions asked participants how they defined the problem. Obviously, this question is related to what they did on Level 1 because defining the problem helps designer to see the big picture. Engineer dyads considered many more factors in this stage than student dyads. Engineer dyads defined the problem by thinking about both the problem and their client's needs. All of them made sure the design met requirements from Americans with Disabilities Act (ADA). At the same time they considered safety issues, aesthetic issues, maintenances of the device, implementation, 
and cost. Some of them even considered the noises generated by the device because the device would be used in a nursing home where a quiet environment is preferred. Some of them thought about making a model of their design so it could be manufactured. The following is the answer from a dyad of engineers:

How did we define the problem? I would say the problem was listed on the paper. It was to create a device to assist elderly people at raising a difficult window. The problem becomes..... I mean there are lots of aspects of the problem. We talked about some safety aspects, making sure that it was safe. One of our initial ideas would cause the blockage of the window, which could cause a safety issue - not being able to escape the window. So that was part of the project definition. We assumed that you need to be able to retrofit and install on an existing building was 65 years old.

So we tried to make it minimally ...... no major construction required. We also assumed that because it is in a nursing home you have to comply with American Disability Act even thought that wasn't stated in the problem.....

When freshman dyads defined the problem, the focus was to assist opening the window. Two dyads mentioned clients of the design. A few dyads talked about the ADA 
while most of them ignored ADA standards though ADA was provided as one of the resources. The following was the answer from a dyad of freshmen:

"I think we both think delivering the force."

"Yes, the problem was that the force that necessary to lift the window was greater that the force that was being applied so we looked at the force required to be supplied and we formulated a solution that would help assisting and increasing the force or giving the force that was applied the mechanical advantage."

Most senior dyads focused on the device itself though they did better than freshman dyads. Most of them were aware of ADA standards, and a few dyads mentioned cost effectiveness as one of their criteria. The following was the answer from a dyad of seniors:

"Base on the disability regulations that we read on the website, we had determined that it had to be a certain amount of weight that we had to reduce, oh, force, to lift the window. This was how we defined the problem."

"Yes, so we said that..... the problem stated that the window was set to be regulated certain weight or force that was required for the..... for those who disabled and we wanted to design something that could reduce the force required to open the windows." 
Participants' sketches supported findings from interviews. In their sketches it was found that, engineer dyads listed more criteria than senior and freshman dyads. Some engineer dyads provided benefits and features of the device. All of engineer dyads' design proposals included the cost of materials while few of student dyads involved cost into the design proposal.

\section{Research Question 3}

The third research question was, "what mental activities are used by engineering experts, seniors, and freshmen when they approach different 'levels of the problem'?" In order to answer this question, mean and standard deviation of FBS codes on different level were calculated across the three types of dyads. The results are shown in Table 12. To be noticed, the data of $\mathrm{F}$ on Level 2 are too small to generate any informative findings hence the results of $\mathrm{F}$ on Level 2 are not discussed. The different use of $\mathrm{F}, \mathrm{B}$, and $\mathrm{S}$ among engineer experts, engineering seniors, and engineering freshmen showed their different design thinking processes.

From Table 12, on Level 1, freshmen, seniors, and engineers all focused most mental activity on Function. The least used mental activity was Behavior. On Level 2, the most used mental activity by freshmen, seniors, and engineers was Behavior while the least used mental activity was Function. On Level 3, the most used mental activity by 
freshmen, seniors, and engineers was Structure and the least used mental activity was

Function. To further explore the answer to the third research question, statistical

techniques were used to compare mean scores from different types of dyads on different

levels.

Table 12

Means and Standard Deviations of FBS on Different Levels of the Problem

\begin{tabular}{|c|c|c|c|c|c|c|c|}
\hline \multirow[t]{2}{*}{$\begin{array}{l}\text { Level of the } \\
\text { Problem }\end{array}$} & \multirow[t]{2}{*}{$\begin{array}{l}\text { FBS } \\
\text { ontology }\end{array}$} & \multicolumn{2}{|c|}{$\begin{array}{l}\text { Freshmen } \\
(\mathrm{N}=10)\end{array}$} & \multicolumn{2}{|c|}{$\begin{array}{l}\text { Seniors } \\
(\mathrm{N}=10)\end{array}$} & \multicolumn{2}{|c|}{$\begin{array}{l}\text { Engineers } \\
(\mathrm{N}=5)\end{array}$} \\
\hline & & Mean & SD & Mean & SD & Mean & SD \\
\hline \multirow[t]{3}{*}{1} & $\mathrm{~F}$ & 4.73 & 1.90 & 4.97 & 1.50 & 10.49 & 7.84 \\
\hline & B & 2.81 & 1.65 & 3.16 & 1.76 & 3.70 & 3.89 \\
\hline & $S$ & 3.47 & .97 & 4.16 & 2.20 & 6.72 & 2.60 \\
\hline \multirow[t]{2}{*}{2} & B & 9.51 & 3.76 & 10.04 & 3.14 & 13.42 & 4.91 \\
\hline & $S$ & 7.74 & 2.20 & 6.54 & 3.11 & 8.19 & 3.63 \\
\hline \multirow[t]{3}{*}{3} & $\mathrm{~F}$ & 1.55 & 1.18 & 1.40 & .79 & 1.76 & 1.37 \\
\hline & B & 19.40 & 3.75 & 23.01 & 6.22 & 14.84 & 4.22 \\
\hline & $\bar{S}$ & 50.38 & 5.88 & 46.26 & 7.58 & 38.71 & 11.78 \\
\hline
\end{tabular}

Before running statistical tests, the normality of data was tested. Based on the

results of Shapiro-Wilk test, most data were normally distributed, except for Function on

Level 3 of engineer dyads, Function on Level 2 of freshman dyads, , and Structure on

Level 3 of engineer dyads. When the comparison involved any of non-normally 
distributed dyads, the researcher used non parametric statistics, instead of parametric statistics. When none of above dyads was involved, the researcher used parametric statistics.

Level 1 represents the designer considering the problem as an integral whole. Results of comparisons between FBS are shown in Table 13. Statistically significant differences in using Function were found between engineer dyads and freshman dyads, as well as between engineer dyads and senior dyads. This result showed that when considering the problem as an integral whole, engineer dyads consider the Function aspect of design more than freshman dyads and senior dyads. In using Behavior, no statistically significant difference was found, which means freshmen and seniors used Behavior as much as engineers when they considered the problem as an integral whole. In the aspect of using Structure, there is a significant different between freshman dyads and engineer dyads. It indicates that engineers used more Structure than freshmen on Level 1. The $p$ value of comparison between seniors and engineers is very small, but not enough to conclude that there is a significant difference between engineers and seniors. Seniors used Structure as much as engineers and freshmen. 
Table 13

Dyad Comparisons (p-value) of FBS on Level 1

\begin{tabular}{ccccccc}
\hline Dyads Compared & \multicolumn{2}{c}{$\mathrm{F}$} & \multicolumn{2}{c}{$\mathrm{B}$} & \multicolumn{2}{c}{$\mathrm{S}$} \\
\cline { 2 - 7 } & $p$ Value & $\begin{array}{c}\text { Effect } \\
\text { Size }\end{array}$ & $\begin{array}{c}p \\
\text { Value }\end{array}$ & $\begin{array}{c}\text { Effect } \\
\text { Size }\end{array}$ & $p$ Value & $\begin{array}{c}\text { Effect } \\
\text { Size }\end{array}$ \\
\hline Freshmen vs. Seniors & .761 & N/A & .650 & N/A & .371 & N/A \\
Freshmen vs. Engineers & $.020^{*}$ & 1.01 & .535 & N/A & $.003 * *$ & 1.66 \\
Seniors vs. Engineers & $.014 *$ & 0.98 & .712 & N/A & .067 & N/A \\
\hline$* b \leq 0.05$ & & & & & & \\
$* * b \leq 0.01$ & & & & & &
\end{tabular}

On Level 2 designers consider interactions between subsystems. Results of

comparisons between FBS on this level are shown in Table 14. No statistically significant

difference was found between any two types of dyads. This result showed that when

designers consider interactions between subsystems, freshman dyads, senior dyads, and

engineering dyads used same amount of Behavior and Structure.

Table 14

Dyad Comparisons (p-value) of FBS on Level 2

\begin{tabular}{lcccc}
\hline Dyads Compared & \multicolumn{2}{c}{ B } & \multicolumn{2}{c}{$\mathrm{S}$} \\
\cline { 2 - 5 } & $p$ Value & $\begin{array}{c}\text { Effect } \\
\text { Size }\end{array}$ & $p$ Value & $\begin{array}{c}\text { Effect } \\
\text { Size }\end{array}$ \\
\hline Freshmen vs. Seniors & .738 & N/A & .331 & N/A \\
Freshmen vs. Engineers & .109 & N/A & .770 & 1.66 \\
Seniors vs. Engineers & .126 & N/A & .375 & N/A \\
\hline
\end{tabular}


On Level 3, designers consider details of subsystems. Results of comparisons

between FBS on this level are shown in Table 15. No statistically significant difference was found in using Function, which means freshman dyads, senior dyads, and engineering dyads used same amount of Function on this level.

Table 15

Dyad Comparisons (p-value) of FBS on Level 3

\begin{tabular}{lcccccc}
\hline Dyads Compared & \multicolumn{2}{c}{$\mathrm{F}$} & \multicolumn{2}{c}{$\mathrm{B}$} & \multicolumn{2}{c}{$\mathrm{S}$} \\
\cline { 2 - 7 } & $p$ Value & $\begin{array}{c}\text { Effect } \\
\text { Size }\end{array}$ & $\begin{array}{c}p \\
\text { Value }\end{array}$ & $\begin{array}{c}\text { Effect } \\
\text { Size }\end{array}$ & $p$ Value & $\begin{array}{c}\text { Effect } \\
\text { Size }\end{array}$ \\
\hline Freshmen vs. Seniors & .748 & N/A & .133 & N/A & .191 & N/A \\
Freshmen vs. Engineers & .759 & N/A & .053 & N/A & $.037^{*}$ & 1.25 \\
Seniors vs. Engineers & .523 & N/A & $.021^{*}$ & 1.54 & .327 & N/A \\
\hline
\end{tabular}

$* b \leq 0.05$

When using Behavior, engineer dyads used it less than senior dyads and freshman

dyads. No significant differences were found between freshman dyads and engineer

dyads, as well as between freshman dyads and senior dyads. A significant difference was

found between senior dyads and engineer dyads. This means engineer dyads used less

Behavior than senior dyads.

When using Structure, no significant differences were found between senior

dyads and engineer dyads, as well as between senior dyads and freshman dyads. This 
means senior dyads and engineer dyads, as well as senior dyads and freshman dyads used same amount of Structure on this level. A significant difference was found between engineer dyads and freshman dyads, which means engineer dyads used less Structure on this level than freshman dyads.

In the interview, there was no question directly related to the research question:

"what mental activities are used by engineering experts, seniors, and freshmen when they approach different levels of the problem?" It was found that when asked what difficulties they had in solving the problem, students' answers were different from engineers'. Most students pointed out that the design challenge did not provide specific numbers that they could use to solve the problem. They needed the size and the weight of the window, the friction coefficient, how much room there was and information like that to quantify their design. Most numbers were related to Structure on Level 3. On the other hands, engineers did not express the urgent need of numbers. They wanted to know more about the clients, conditions in the nursing home, and other related information. This result indicates that engineer experts are used to solve ill-structured problems and they tended to pay more attention to non-technical variables that technical variables.

When participants realized specifications were not provided, students and engineers had different solutions. From their sketches we could see, student participants, 
especially freshman participants, tended to assume specific numbers based on their experiences. For example, they would assume the window is 3 feet tall and 3 feet wide, and the weight of the window is 20 pounds. Engineers usually made their design flexible so their product could be used on windows have different sizes. The choice of different solutions indicated that students highly relied on Structure to finish the design challenge.

\section{Other Themes and Phenomenon Found from Qualitative Data}

\section{Alternative Solutions and Time Management in Engineering Design}

It was recommended to all the dyads in this study to finish their design in an hour.

The actual time spent by each dyad varied. The range of time spent by engineer dyads to complete the design was 50 to 67 minutes while it was 39 to 98 minutes for student dyads. In terms of completing the design within recommended time, engineer dyads managed time better than student dyads. When too little time was spent to finish the design, designers had wasted the time which they could have used to improve their design. In the interviews it was found that two dyads only generated one alternative solution through the design process, and one of the dyads completely finished the design in 39 minutes. Another two dyads spent 98 and 93 minutes to finish the design. We found they both spent a majority of time analyzing different solutions. One of them admitted they "wasted too much time on those possible solutions" because they had "too many ideas to decide." 
Engineer dyads were aware of the limitation of time. When talked about alternative solutions, they mentioned that they "should spend some time on it, but not too much." All of engineer dyads generated more than one alternative solution in the process of design. They also mentioned that design ideas should not be eliminated too early without analysis because it might develop into a good solution. Engineers believed that they should reevaluate their solutions through the entire design process to get better results.

When participants were asked why and how they chose their final solution, most students' answers were "simple" and "easy," including "simple to use," "easy to maintain," and "simplest one we can come up with." Engineers provided answers that were different from students. They believed there were many methods to solve the problem and "you need to decide which can make it works best, which way you feel more comfortable with." They were also "open to combine ideas." For some student dyads, they expressed difficulties they had in choosing the final solution.

\section{Cost in Engineering Design}

Cost is an important issue in engineering design. In the interview, participants were asked how they defined the problem. Engineers emphasized the importance of cost by repeatedly mentioning "cost effectiveness." They also incorporated the cost as one of their criteria, because when the cost of the device is too high the nursing home should 
just replace the window. A dyad of engineer provided a specific number of how much the device they designed would cost.

Senior students paid attention to the cost issue. They did not explore it at the level of the engineer dyads. Some dyads did better than others when dealing with the cost. A few of them mentioned "cost effective" in interviews and some dyads did not consider this issue at all. One dyad admitted that they did believe cost was important but they did not know the cost of materials.

Freshman dyads considered cost issues the least. Only a few dyads considered this aspect in defining the problem. Most of them did not realize that the cost of the entire design should be less than simply replacing the window.

\section{Communication}

In the interview, when asked about difficulties in the design process, one of the freshmen dyads expressed their difficulties in communication when exchanging ideas. An example of this difficulty is presented below.

"Communication, because he (his partner) would have an idea and I know he (his partner) is doing his best to describe it and I am not understanding it. And I have an idea and I am terrible at describing it. I know it. And I know I am not conveyed to him (his partner). So I am trying to picture something and I am struggling with that and I cannot 
get it crossed to him (his partner). So that was why we were drawing. We were trying. I was trying desperately to, to be able to communicate my idea and I was trying desperately to grab his (his partner's) idea."

A senior dyad also had a communication issue. They had disagreements on which solution to choose. They spent a lot of time on convincing each other, which caused them to go beyond the time limit. On the other hand, communication was not an issue for engineer dyads. In the interview, when asked what difficulties they met, none of the engineer dyads mentioned communication related issues. They showed their communication ability by brainstorming ideas, applying shared standards in choosing the final solution, and working on two different tasks at the same time.

\section{Textbook Problems and Real World Problems}

In the interview with a couple of freshman dyads, students explicitly pointed out differences between textbook problems and real-world problems:

"Umm..,... to be honest, my biggest difficulty was..... like in all of our classes we have taken, at least for me, they gave us every variable we need, but they do not give us actual variables. And we know everything we need to know. In this one (the design challenge), there are so many wild things. We don't know what's needed what's not 
needed. That was the biggest problem for me. It's hard because I have always been told what I need to know."

"In our class we are given seven pieces, and you need three of them or four of them to actually solve. But you have every piece of information you need. And in this one (the design challenge), we are kind of given... we are given a lot of pieces, and we are not given a lot of pieces at the same time. There was a lot of data that we could go through and read, but we could not go through and read every line of the taken notes and those websites, but a lot of it isn't applicable and a lot of it isn't complete."

“That's one of my hardest problems because I didn't... I thought I was pretty good, to be honest, at homework because, I'm like 'oh, I can do this'. But when I actually doing a problem, I realize that it's not as easy as it's on the homework. And it's not even close...."

Senior dyads did not express difficulties in this aspect as much as freshman dyads because they had taken or were taking senior design course in the time of the study. They had some experiences with open-ended problems in their design courses. A few of them indicated that they had difficulties in realizing their ideas and they were not capable of "building a product." 


\section{Summary}

This research collected both quantitative and qualitative data. The analysis of quantitative data used statistical techniques and the analysis of qualitative data used the constant comparative method. The results of analyzing both quantitative data and qualitative data helped answer the research questions. In addition, the analysis of qualitative data allowed some themes to emerge.

The results showed that students' and engineers' design thinking process were different. To be more specific, students were different from engineers in using problem decomposition/recomposition; students and engineers spent their cognitive effort

differently; and students and engineers used different mental activities when they approached different levels of the problem. A series of implications and recommendations were made based on the results which are further discussed in chapter V. 


\section{CHAPTER V}

\section{DISCUSSIONS, IMPLICATIONS, AND RECOMMENDATIONS}

Engineering design is a core of engineering education and in this study, engineering design was investigated. The focus of the study was the use of problem decomposition and problem recomposition among engineering freshmen, engineering seniors, and engineering experts. They worked in dyads to complete the same engineering design challenge. Their conversation and behavior was video and audio recorded. Two coding systems including FBS framework and "levels of the problem" were used to analyze data. In addition, participants' interviews and sketches worked as qualitative data to help answer research questions.

One of the assumptions of this research is engineering experts are more skilled than college students in engineering design. With this premise, the researcher compared engineering experts' design behavior with freshmen's and seniors' to see how students design skills aligned with experts'. The results of the study could shorten the transition between novices and experts in engineering design. 


\section{Discussions}

\section{Research Question 1}

The percentages of using problem decomposition and recomposition across three types of dyads were calculated. In order to examine if there were statistically significant differences, the researcher ran a series of statistical tests. The results showed that engineer dyads used problem decomposition and problem recomposition more than senior dyads and freshmen dyads. Qualitative data from interview also support this result. Ho (2001) studied the use of problem decomposition and recomposition between an expert and a novice. The settings of this study and Ho's study were different. In Ho's study, the participants worked as individuals while in this study participants worked in dyads. The design challenge in Ho's study was a complex electrical engineering design problem while in this study the challenge was the window-opener design. In addition, the novice in Ho's study was a master student and in this study the undergraduate student was the novice. In spite of differences in research settings, the results of this study are consistent with Ho's. Both studies suggested that there is a gap in using problem decomposition/recomposition between experts and novices. 
As mentioned earlier, problem decomposition and problem recomposition are

commonly used strategies by professional engineers. In fact, in interviews with engineer participants, they emphasized the importance repeatedly,

"(We use problem decomposition and recomposition) all the time. Almost a hundred percent of the time because usually the problem is so... so big that you cannot tackle them as a whole, you have to break them down."

"I think certainly. Spontaneously you need to break things down. In my experience, I usually like to start with an overall. You can look at the whole project as a whole. Go through it, break it into the... various pieces you can break into. Sometimes you start with a big piece, sometimes you start with a difficult piece. Work through those pieces, and then at the end I'd like to look at it as a whole again...."

While problem decomposition and recomposition is such a crucial strategy in engineering design, the results of this study showed that there was no difference between freshman dyads and senior dyads using this strategy in engineering design. It would suggest that engineering education does not place enough importance on problem decomposition and problem recomposition. Throughout the engineering program, students do not learn adequate knowledge about using problem decomposition and problem recomposition in engineering design, hence students in the first year of the 
engineering program performed similar to students about to finish the engineering program.

\section{Research Question 2}

Percentages of codes on different levels of the problem were calculated to answer research question 2. The results of the study showed engineer dyads, senior dyads, and freshmen dyads all spent most of their cognitive effort on Level 3 and the least of their cognitive effort on Level 1. The researcher ran a series of statistical tests to further investigate cognitive effort spent on different levels of the problem. Qualitative data from interviews and sketches also helped answer this question. The quantitative data showed that on Level 1, engineer dyads spent more cognitive effort than senior dyads and freshman dyads. On Level 2, engineer dyads spent more cognitive effort than senior dyads and freshman dyads. On Level 3, engineer dyads spent less cognitive effort than senior dyads and freshman dyads.

Past studies identified two types of problem decomposition: breadth-first approach and depth-first approach. The breadth-first decomposition focuses on exploring various solutions of each sub-problem and avoids deep exploration to any specific solution in the early stage while depth-first decomposition tends to explore a specific subproblem in detail before other sub-problems are investigated (Ormerod \& Ridgway, 
1999). In this research, Level 3 represent designers considering details of sub-problems.

Student dyads spent more cognitive effort on this level because most of them used depthfirst decomposition and spent a majority of cognitive effort exploring details of a certain sub-problem. Engineer dyads used breadth-first approach. Unlike student dyads, the distribution of their cognitive effort was more balanced across three levels of the problem than students'.

Among student dyads, the distribution of cognitive effort of senior dyads was more aligned with engineer dyads. The distribution of their cognitive effort was more balanced than freshman dyads. However, statistically significant differences were found on all levels between engineer dyads and senior.

This finding is consistent with previous studies. In 1981, Jeffries and colleagues found that novices were prone to using depth-first decomposition in problem solving while experts were more inclined to use breadth-first decomposition (Jeffries et al., 1981). Ball and colleagues (1994) had similar findings in their research in which the individual design behavior was studied in the context of electrical engineering. Ormerod and Ridgway (1999) conducted two experiments in 1999 and they reached similar conclusions. Ho (2001) further confirmed this conclusion in his study in 2001. 


\section{Research Question 3}

The percentages of using F, B, and S on each level were calculated. Statistical techniques were used to further compare if there were differences among engineer dyads, senior dyads, and freshman dyads.

On Level 1, designer considered the problem as an integral whole. Engineer dyads were found to use more Function than senior dyads and freshmen dyads. Function is related to the purpose of design and is the expression of requirements, hence Function on Level 1 represents the definition of the problem. The quantitative data showed that there were statistically significances between engineer dyads and student dyads in using Function on Level 1. With findings from qualitative data, we can conclude that there is a gap in defining the problem between engineer dyads and student dyads.

Engineer dyads considered many more aspects of the design than seniors and freshman did in defining the problem. Engineer dyads defined the problem by thinking about both the problem and their client's needs. They considered Americans with Disabilities Act (ADA), safety issues, aesthetic issues, maintenances of the device, implementation, cost, noises generated by the device, and manufacture issues. When freshman dyads defined the problem, the focus was to assist opening the window. Senior 
dyads also focused on the device itself but they were aware of ADA standards and a few senior dyads mentioned cost effectiveness as one of their criteria.

This result is consistent with previous studies. Atman colleagues found that experts tended to spend more time on problem definition than novices (Atman et al., 2007). Jain and Sobek (2006) reached a similar conclusion in their research in 2006.

Novices do not spend enough time on problem definition because they do not realize that they do not completely understand the problem (Atman et al., 2007).

No differences were found in the use of Behavior on Level 1. B contained two aspects, Be and Bs. Be is the behavior that designers expected to have and Bs is the behavior derived from the design. The results indicated that when considering the problem as an integral whole, engineer dyads, senior dyads, and freshman dyads spent the same amount of time on the behavior of the design.

On Level 1, engineer dyads used more Structure than senior dyads and freshman dyads. Structure describes the components of objects or systems and their relationships. No difference was found between senior dyads and freshman dyads. The results illustrated that when considering the problem as an integral whole, engineer dyads tended to pay more attention to the object or system and the relationship between them. 
On Level 2, no differences were found in using Function, Behavior, and Structure.

The results illustrated that when considering interactions between subsystems, engineer dyads, senior dyads, and freshman dyads used similar mental activities. On Level 3, designers considered details of subsystems. Function was rarely used by all dyads on this level and no differences were found in using Function among three types of dyads.

Engineer dyads were found to use less Structure than freshman dyads. Senior dyads' use of Structure was more aligned with engineer dyads'. No difference was found between senior dyads and engineer dyads. In addition, no difference was found between senior dyads and freshman dyads. Engineer dyads were found to use less Behavior than senior dyads but there was no difference between engineer dyads and freshman dyads. In addition, no significant difference was found between freshman dyads and senior dyads. From Research Question 2 it was found that student dyads tended to spend more cognitive effort on Level 3 because they tended to use depth-first decomposition. The results of Research Question 3 on Level 3 showed that when considering details of subsystems, freshman dyads tended to use more Structure while senior dyads tended to use more Behavior. The results can be explained by engineering curriculum. In the beginning of most engineering programs, students take foundational mathematical and scientific courses such as calculus. In the second year of the engineering curriculum, 
students start learning advanced mathematical and scientific courses such as dynamics and statics. These courses teach students to work with technical variables including power, weight, height, time, etc. Technical variables are directly related to Structure hence freshman dyads used it more. In the second half of engineering programs, students take engineering design courses which teach them to better analyze the design problem. Students take their senior design course at the end of their programs. Students in senior dyads already had the knowledge to work with technical variables hence their analysis tended to be more in-depth. They used more Behavior which deals with the expected behavior from the design or the behavior from structures.

When examining the results of research question 3, student dyads, especially freshman student dyads, considered more Structure than engineer dyads. On Level 1, when designers considered the problem as an integral whole, engineer dyads considered more Function than student dyads. Requirements (R) were not coded by "levels of the problem" because it refers to the design requirements but $\mathrm{R}$ was expressed through $\mathrm{F}$. The transfer from $\mathrm{R}$ to $\mathrm{F}$ and from $\mathrm{F}$ to $\mathrm{Be}$ represent the process of formulation, which set the foundation of the entire design. Students used less F on Level 1 than engineers, which means they did not understand the problem as well as the engineers did. However, they 
spend more of their energy and cognitive effort on the design itself (S) on Level 1 and

Level 3.

This result can be interpreted by the existing engineering curriculum. During the first two years of most engineering programs, students take mathematical and scientific courses. These courses teach students how to find solution to problems which focus on design (S) aspect. Courses like this are defined as "foundational courses" of engineering programs. Understanding of the problem is the basis of engineering design because a design is never successful without understanding the clients' needs and requirements.

\section{Discussion of Findings from Qualitative Data}

A series of interesting findings emerged from interviews and analyzing participants' sketches. In the process of generating alternative solutions, student dyads tended to generate too many or too few solutions compared with engineering dyads. This caused the time management issue. Some dyads only generated one solution, they

finished their design at a premature stage. They did not make use of the time which they could have used to optimize their design. When examining engineering curriculum, we find that in most courses, students are taught to generate only one solution instead of multiple ones. It also explains why some dyads only generated a solution through the entire design. For those dyads who generated too many alternative solutions, they spent a 
lot of time analyzing solutions, which lead them to either go way beyond the time

limitation, or haphazardly select a final solution in the end of the design period.

Fricke (1996) had similar findings in a study conducted in 1996. He found experts tended to use balanced searching strategy, which generated an appropriate number of solutions. Novices generated either too few solutions or too many solutions. When few alternatives were available, they tend to be fixated on the solution stage too early to have access to enough information. When there were too many alternative solutions, cognitive overload was generated and they tended to spend their time and energy on managing and organizing solutions instead of evaluating and improving solutions (Fricke, 1996).

Problem definition is the first and foundation-setting stage of engineering design.

As mentioned earlier, student dyads in this study were found to show many weaknesses in this stage. In analyzing qualitative data, student dyads showed their inadequacy in managing cost issue in the design. The cost issue is a crucial content in the design challenge because if the cost of their design is more expensive than replacing the old window, the problem itself will be eliminated. Unfortunately, most students dyads, especially freshman, did not realize this issue. In fact, some of them did not consider the cost of the device at all. Students tended to show their inadequacy in problem definition 
by spending less time and energy on this stage, which was consistent with results from previous studies (Atman et al., 2007; Jain \& Sobek, 2006).

In analyzing qualitative data, engineers were found more comfortable working in groups than students. Student dyads had various difficulties when they worked together. A freshman dyad of students expressed their inadequacy in understanding each other's ideas. Another freshman dyad of students had disagreements about which final solution to choose, which cost them a lot of time. A few senior dyads pointed out that they did not make good use of their time by work individually on different tasks at the same time.

In the first two years of most engineering programs, students take engineering foundational courses, such as calculus, dynamics, and statics. A majority of the time they work as individuals in those courses. Most of them lack experiences in working in groups. This may be the main reason why freshman dyads in this study showed their weakness when working with others. Most engineering programs provide more opportunities to practice working in groups later in the program, which explained why senior dyads performed better than freshman dyads when it comes to work in groups. However, the performances of senior dyads were still very different from engineer dyads'. The engineering program did not fully prepare students with skills to work in groups. This 
finding is consistent with a series of previous studies (Meier, Williams and Humphreys,

2000; Sageev \& Romanowski, 2001; Scott and Yates, 2002; Holcombe, 2003).

In engineering programs, when engineering students work in groups, the partners

they worked with are their classmates. Their classmates' age, major, and background are

usually similar to themselves. The problems engineering students typically solve when

they work in groups, are laboratory assignments and homework. The most

comprehensive project they encounter is their senior design project. Students meet

several times to complete this project. On the other hand, professional engineers always

work with people from diverse backgrounds, sometimes they even work with people who speak different languages. Professional engineers work in large groups all the time. They typically solve extremely complicated problems which require them to work together every day for extended period. In addition, studies (Cross \& Cross, 1995; Olson \& Olson, 2000; Zolin, Hinds, Fruchter, \& Levitt, 2004) have shown that factors such as trust, social skills, and common ground, affect behaviors of group design. The performance of group design is a complex result of the above factors, and is more than the sum of performances from each group member (Kan \& Gero, 2009). The size and the diversity of groups, the complexity of problems, and the amount of accumulated experience explained why 
freshman dyads, senior dyads, and engineer dyads performed differently when working in groups.

\section{Implications}

Student participants of the study were all from the College of Engineering at Utah State University and engineer participants were all from engineering companies in Utah. The findings of the study may vary when applied on other conditions.

\section{Implications for Engineering Education}

Engineering design has always been a significant content area in engineering

education. Problem decomposition/recomposition is a frequently used strategy by

professional engineers. The results of this study showed that there is a gap between

engineering students and engineering experts in using problem decomposition and

problem recomposition. In addition, no differences were found between engineering

freshmen and engineering seniors, which indicates that engineering education has spent

little time teaching problem decomposition and problem recomposition. In order to better

prepare students for future careers, it is extremely important to incorporate this content into engineering education. There is a need to develop supplemental teaching materials featuring problem decomposition and problem recomposition. 
In the interview, students constantly mentioned that the design challenge was different from their "textbook problems." Both freshmen dyads and senior dyads expressed their frustrations when they found the specifications of the window were not provided. However, ill-structured problems are commonly seen in real life. One of the goals of engineering education is to teach students to solve real-world problems.

Currently, students are trained to solve formula-based problems more than practical real life problems. As previously mentioned, engineering majors have a higher drop-out rate in the first and second year compared with other fields. This result implies that engineering education should introduce more real-world design problems to engineering students in an early stage. Research showed that this kind of activities help student better learn scientific and mathematical courses (Soloway, Guzdial, \& Hay, 1994). By better learning those foundational courses, students who would have dropped out because of the difficulties they meet in scientific and mathematical courses will be retained. In addition, incorporating more real world contexts is an effective way to increase the enrollment of females (Du \& Kolmos, 2009), which will help retain females students in engineering programs.

Problem definition is the first stage of engineering design. This study found that students showed their weakness in this stage compared with engineering experts. This 
conclusion is consistent with previous studies (Atman et al., 2007; Jain \& Sobek, 2006).

In the first two years of most engineering programs, students learn mathematical and scientific courses. Those theories and scientific principles help students solve problems instead of defining problems. It also implies that more real world problems should be incorporated into the early stage of engineering programs. When solving textbook problems, students spend little time defining them because those problems are wellstructured. By exposing students to ill-structured real world problems, they will become aware of the importance of problem definition. At the same time, senior dyads were also found to poorly define the problem, which implies that engineering education should place more importance on teaching problem definition in general.

\section{Implications for Engineering Curriculum}

This study found that students lacked the skill of working with others, which implies to provide more opportunities for students to work in groups in engineering curriculum. ABET (ABET, 2008) recognized teamwork as a crucial skill for engineering students and the key to productivity (Mendelsahn, 1998). Employers also identify teamwork skills as an important quality for engineering graduates to have (Smith, 2008). In this study, students did not show enough competence when working in groups compared with experts. On the other hand, when students start working in industry, they 
have to collaborate with other engineers on a daily basis. This implies that engineering curriculum has not completely prepared students for working in groups. In the first two years of most engineering curriculum, students take foundational engineering courses, most of which are scientific and mathematical courses. When taking these courses, students spend most of the time working as individuals. In order to better prepare students for their future jobs, engineering curriculum should engage students in group activities from the beginning of the program. More group activities such as group discussion, group presentation, and group study should be incorporated in mathematical and scientific courses in which students traditionally work as individuals. At the same time, engineering curriculum should increase the proportion of group work. By spending more time working in groups, students can learn how to express their ideas as well as make compromises, which will help them success when they work as engineers in the future.

In this study, many of the students were not comfortable during the engineering design challenge because it was open-ended and very different from their textbook problems, especially freshman students. This implies that engineering curriculum should include more real-world problems in all courses. For those courses that it is difficult to 
incorporate real-world problems, introducing the applications of the theory might help as

well. Students will not be so overwhelmed when they face open-ended problems.

\section{Implications for Engineering Educators}

The study found engineering students, especially engineering freshmen lack

abilities to work with open-ended problems. It implies that engineering educators should

bring scenarios of engineering design to students as much as possible. More importance

should be placed on engineering design strategies in their classrooms, especially

strategies that are commonly used in industry. Educators should help students see the

connections between theories and their real life, which is extremely important in basic

engineering courses. In most of those courses, students only learn theories of science and

mathematics. Introducing the application of the theory and connecting theories with

concepts of real life can help students better learn equations and formula that are too

abstract to understand. It helps increase students' interests as well. Educators should

continue to share their own design experiences as well as invite guest speakers to help

students understand how it is like to work as engineers.

Engineering educators should provide students more opportunities to work

dynamically in groups. Educators should teach students to express their ideas as well as

make compromises when working with other people. Educators should assigning students 
into groups instead of letting students choose who to work with because in industry,

engineers do not decide who to work with. When assigning groups, educators should encourage students who have different genders, ages, and races to work together so they

can learn how to work with people from diverse backgrounds. At the same time,

educators should rotate students in different groups so they can work with different

people. Working with different people will help students adapt to dynamics in groups and get along with group members who have different personalities. In addition, it can teach students to respect each other as well as be open-minded to new perspectives and opinions.

\section{Recommendations for Future Research}

From the finding of the research, several recommendations can be made. The first recommendation for the future study is to conduct a similar study in multiple locations. In this study, all student participants were from Utah State University and all engineer participants were from engineering companies in Salt Lake City and Cache Valley, therefore, the generalizability of the study may not be nationwide. Future research should consider carrying this study in states on east coast, west coast, and mid-west.

Another recommendation is to incorporate more participants from minority groups. Most participants $(80 \%)$ in the study were white male, which means minority 
population was poorly represented. Engaging more minority participants will also help improve the generalizability of the results.

The third recommendation to future studies is to increase the sample size. This study had a decent sample size. The larger sample size is, the higher the power will be. Considering the coding process of the study was time consuming, an extremely large sample size will create a huge workload. It is recommended that each type of groups contains at least 20 groups in the future study. This will help increase the power of the study and improve the generalizability of the results.

The fourth recommendation is to investigate differences between genders. It would be interesting to study if there are any different thinking patterns or design strategies used between males and females. Engineering educators can design learning materials or change existing curriculum to make engineering education more "femalefriendly" since the field needs more females (Ihsen, 2005) and engineering has one of the lowest participation rates of women across all professions (Lewis, Harris, \& Cox, 2007). Another recommendation is to compare novices and experts' engineering design behavior for a longer period of time. In this study, participants were recommended to finish the entire design within an hour. They submitted a design proposal to present their design ideas as the final product and they did not actually test or build their design. In 
future studies, it is recommended to ask participants to finish the entire design process similar to what engineers do in the real world. The results might be more informative. In addition, the process of testing ideas and building product might provide useful information that was not found in this study.

It is also recommended to use a more complicated design challenge in future study. This study did not use a complex engineering design challenge because engineering freshmen were involved. The difficulty of a complex engineering design challenge might be beyond their abilities. In Ho's (2001) study he asked participants to design "a communication facility with answering function" (p. 30). It was a complex electronic engineering design challenge and participants were all electronic engineer. In future studies, when focusing a certain field of engineering, researchers are recommended to use more complex design challenges to study more complicated thinking process.

As mentioned earlier, problem decomposition and problem recomposition were found to be poorly taught in engineering education. More specifically, learning problem decomposition and problem recomposition should be included in Engineering Certification Test. The reason is there is much content to cover in engineering curriculum, adding them into the test can highlight the importance of problem decomposition and problem recomposition as well as arouse students' attention to this content. At the same 
time, it inspires researchers and educators to develop new teaching materials or models to address this problem. The teaching materials could be supplemental materials which featuring problem decomposition and problem recomposition and should be easily incorporated in a wide range of engineering programs. Workshops are also helpful in preparing engineering educators in teaching problem decomposition and problem recomposition. Another option is providing an example of engineering design featuring the use of problem decomposition and problem recomposition in the format of a video clip or a presentation. It assist students understand the concept of problem decomposition and problem recomposition easily and quickly. It will be fascinating to see what teaching material and models are developed and how they improve students' design skills in future studies. 


\section{REFERENCES}

ABET. (2007). ABET 2009 requirements. Retrieved from http://www.abet.org/Linked\%20DocumentsUPDATE/Criteria\%20and\%20PP/E001\%2008-09\%20EAC\%20Criteria\%2011-8-07.pdf

ABET. (2008). Criteria for accrediting engineering programs, 2 November 2002. Retrieved from http://www.abet.org/criteria.html

Ahmed, S., Wallace, K. M., \& Blessing, L. M. (2003). Understanding the differences between how novice and experienced designers approach design tasks. Research in Engineering Design, 14(1), 1.

Akin, O . (1986). Psychology of architectural design. London, UK: Pion.

Alexiou, K. (2010). Coordination and emergence in design. Codesign, 6(2), 75-97. doi:10.1080/15710882.2010.493942

American Society for Engineering Education (ASEE). (2004). Proceedings of the American Society for Engineering Education Annual Conference \& Exposition.

Anderson, J R (1983) The architecture of cognition, Cambridge, MA: Harvard University Press.

Atman, C. J., Adams, R. S., Cardella, M. E., Turns, J., Mosborg, S., \& Saleem, J. J. (2007). Engineering design processes: A comparison of students and expert practitioners. Journal of Engineering Education, 96(4), 359 -379.

Atman, C. J., \& Bursic, K. M. (1998). Verbal protocol analysis as a method to document engineering student design processes. Journal of Engineering Education, 87(2), 121-132.

Atman, C. J., Kilgore, D., \& McKenna, A. (2008). Characterizing design learning: A mixed-methods study of engineering designers' use of language. Journal of Engineering Education, 97(2), 309-326.

Arvanitis, T. N., Todd, M. J., Gibb, A. J., Orihashi, E. (2001). Understanding students' problem-solving performance in the context of programming-in-the-small: An 
ethnographic field study. Proceedings of the Frontiers in Education Conference, 2001. 31st Annual - Volume 02, IEEE Computer Society: F1D-20-23 vol.22.

Bainbridge, L. (1999). Verbal reports as evidence of the process operator's knowledge. International Journal of Human- Computer Studies, 51, 213- 238.

Ball, L. J., Evans, J. T., \& Dennis, I. I. (1994). Cognitive processes in engineering design: A longitudinal study. Ergonomics, 37(11), 1753-1786. doi:10.1080/00140139408964950

Ball, L. J., Ormerod, T. C., \& Morley, N. J. (2004). Spontaneous analogizing in engineering design: A comparative analysis of experts and novices. Design Studies, 25(5), 495-508. doi:10.1016/j.destud.2004.05.004

Cardella, M. E., Atman, C. J., Turns, J., \& Adams, R. S. (April 24, 2008). Students with differing design processes as freshmen: Case studies on change. International Journal of Engineering Education, 24, 2, 246-259.

Chandrasekaran, B. (1990). Design problem solving: A task analysis. AI Magazine, 11(4), $59-70$.

Christensen, B., \& Schunn, C. D. (2007). The relationship of analogical distance to analogical function and preinventive structure: The case of engineering design. Memory and Cognition, 35(1), 29-38.

Christiaans, H. H. C. M., \& Dorst, K. H. (1992). Cognitive models in industrial design engineering: A protocol study. Design Theory and Methodology,42, 131-140.

Chrysikou, E. G., \& Weisberg, R. W. (September 01, 2005). Following the wrong footsteps: Fixation effects of pictorial examples in a design problem-solving task. Journal of Experimental Psychology: Learning, Memory, and Cognition, 31, 5, 1134-1148.

Corder, G. W., \& Foreman, D. I. (2009). Nonparametric statistics for non-statisticians: A step by step approach. Hoboken, NJ: Wiley.

Crisp, V. (May 01, 2008). The validity of using verbal protocol analysis to investigate the processes involved in examination marking. Research in Education, 79, 1-12. 
Cross, N. (2002). Creativity cognition in design: Processes of exceptional designers. In T. Hewett \& T. Kavanagh (Eds.), Creativity and cognition (pp. 14-19). New York, NY: ACM Press.

Cross, N. (2004). Expertise in design: An overview. Design Studies, 25(5), 427-441.

Cross, N., \& Cross, A.C. (1995). Observations of teamwork and social processes in design. Design Studies, 16, 143-170.

de Vere, I., Melles, G., \& Kapoor, A. (2010). Product design engineering - a global education trend in multidisciplinary training for creative product design. European Journal of Engineering Education, 35(1), 33-43. doi:10.1080/03043790903312154

Deng, Y. M., \& Zheng, D. D. (2006). Behavioural modelling for design decomposition and design equations derivation. International Journal of Advanced Manufacturing Technology, 29(11/12), 1105-1114. doi:10.1007/s00170-0050011-3

Dorst, K., \& Cross, N. (2001). Creativity in the design process: Co-evolution of problemsolution. Design Studies, 22(5), 425-437. doi:10.1016/S0142-694X(01)00009-6

Draper, S. (2009). Engineering design process. Technology and Children, 13(4), 8-10.

Drew, C. J., Hardman, M. L., \& Hosp, J. L. (2008). Designing and conducting research in education. Thousand Oaks, CA: Sage.

Du, X., \& Kolmos, A. (2009). Increasing the diversity of engineering education: A gender analysis in a PBL context. European Journal of Engineering Education, $34,425-437$.

Dufresne, R., Gerace, W. J., Hardiman, P. T., \& Mestre, J. P. (1992). Constraining novices to perform expert-like problem analyses: Effects on schema acquisition. Journal of the Learning Sciences, 2, 307-331.

Dym, C. L. (1999). Learning engineering: design, languages, and experiences. Journal of Engineering Education, April 1999, 145-148. 
Dym, C. L. (2004). Design, Systems, and Engineering Education. International Journal of Engineering Education, 20(3), 305-312.

Dym, C., Agogino, A., Eris, O., Frey, D., \& Leifer, L. (2005). Engineering design thinking, teaching, and learning. Journal of Engineering Education, 94(1), 103120.

Dym, C. L., Little, P., Orwin, E. J., \& Spjut, R. E. (2009). Engineering design: A projectbased introduction $3^{\text {rd }}$ eds. Hoboken, NJ: Wiley.

Eide, A. R., Jenison, R. D., Mashaw, L. H., \& Northrup, L. L. (2002). Introduction to engineering design and problem solving. Boston, MA: McGraw-Hill.

Egan, D. E., \& Schwartz, B. J. (1979). Chucking in the recall of symbolic drawings. Memory \& Cognition, 7(2), 149-158.

Ericsson, K. A., \& Lehmann, A. C. (1996). Expert and exceptional performance: Evidence of maximal adaptation to task constraints. Annu Rev Psychol 47, 273305.

Ericsson, K. A. \& Simon, H. A. (1993). Protocol analysis: Verbal reports as data. Cambridge, MA: The MIT Press.

Felder, R.M., \& Brent, R. (2007). Cooperative Learning. In P.A. Mabrouk (Eds)., Active learning: Models from the analytical sciences, ACS Symposium Series 970, Chapter 4. Washington, DC: American Chemical Society. On-line at $<$ http://www.ncsu.edu/felder-public/Papers/CLChapter.pdf>. See also <http://www.ncsu.edu/felder-public/Student-Centered.html>.

Fricke, G. (1996). Successful individual approaches in engineering design. Research in Engineering Design, 8, 151-165.

Gall, M., Gall, J., \& Borg, W. (2007). Educational research: An introduction (8th ed.). Boston, MA: Pearson, Allyn and Bacon.

Gericke, K., Schmidt-Kretschmer, M., \& Blessing, L. (2007). Dealing with requirements: inferences on idea generation in the early stages of product development, in Janet McDonnell \& Peter Lloyd (Eds.), DTRS7 Design Meeting Protocols: Workshop Proceedings (pp. 127-140). London. 
Gero, J. S. (1990). Design prototypes: A knowledge representation schema for design. AI Magazine, 11(4), 26-36.

Gero, J. S. (2010). Generalizing design cognition research. In K Dorst (Eds.), DTRS8: Interpreting design thinking, DAB documents, (pp. 187-198). Sydney.

Gero, J. S., Jiang, H., Williams, C. (2013). Design cognition differences when using unstructured, partially structured and structured concept generation creativity techniques, International Journal of Design Creativity and Innovation, 1(4), 196214.

Gero, J. S., \& Kan, J. W. (2009). Learning to collaborate during team designing: Some preliminary results from measurement-based tools. In A. Chakrabarti (Ed.), Research into design (pp. 560-567). Delhi, India: Research Publications.

Gero, J. S., Kan, J. W. T., \& Pourmohamadi, M. (2011). Analysing design protocols: Development of methods and tools. In A. Chakrabarti (Ed), Research into design, (pp. 3-10).

Gero, J. S., \& Kannengiesser, U. (2004). The situated function-behaviour-structure framework. Design Studies, 25(4), 373-391.

Gero, J. S., \& Kannengiesser, U. (2007). An ontology of situated design teams, AIEDAM, 21(4), 379-391.

Gero, J. S., \& Mc Neill, T. (1998). An approach to the analysis of design protocols. Design Studies, 19(1), 21-61.

Glaser, B. (1965). The Constant Comparative Method of Qualitative Analysis. Social Problems, 12(4), 436-445.

Goel, V., \& Pirolli, P. (1989). Motivating the notion of generic design within information process: The design problem space. Artificial Intelligence Magazine, 10(1), 19-36.

Goldschmidt, G. (2003) Cognitive economy in design reasoning. In Lindemann, (Ed.), Human behavior in design, (pp. 53-62). London, UK: Springer Verlag.

Green, A. (1998). Verbal protocol analysis in language testing research: a handbook. Cambridge, UK: Cambridge University Press. 
Guindon, R. (1990). Designing the design process: exploiting opportunistic thoughts. Human-Computer Interaction, 5, 305-344.

Hailey, C. E., Erekson, T. L., Becker, K. H., \& Thomas, M. (2005). National Center for Engineering and Technology Education. The Technology Teacher, 64(5), 23-26.

Hall, G. H., Jackson, M., Lanney, R. C., Nuseibeh, B., \& Rapanotti, L. (2002). Relating software requirements and architectures using problem frames. Requirements Engineering, 2002. Proceedings. IEEE Joint International Conference, pp. 137144. Essen, Germany.

Hmelo-Silver, C. E. (2004). Problem-based Learning: What and how do students learn? Educational Psychology Review, 16(3), 235-266.

Ho, Chun-Heng. (2001). Some phenomena of problem decomposition strategy for design thinking: Differences between novices and experts. Design Studies, 22(1), 27-45.

Hoffman, R. R. (1996). How can expertise be defined? Implications of research from cognitive psychology. In R. Williams, W. Faulkner, \& J. Fleck (Eds.), Exploring expertise (pp. 81-100). Edinburgh, Scotland: University of Edinburgh Press.

Holcombe, M. L. (2003). ET grads -How'd the transition go? 2003 ASEE Annual Conference and Exposition, 20-22 June, 2003, Nashville, TN.

Hughes, J., \& Parkes, S. (2003). Trends in the use of verbal protocol analysis in software engineering research. Behaviour \& Information Technology, 22(2), 127. Retrieved from EBSCOhost.

Ihsen, S. (2005). Special gender studies for engineering? European Journal of Engineering Education, 30, 487-494.

Jain, V. K., \& Sobek, D. K. (2006). Linking design process to customer satisfaction through virtual design of experiments. Research in Engineering Design, 17(2), $59-71$.

Jeffries, R., Turner, A. A., Polson, P. G., \& Atwood, M. E. (1981). The processes involved in designing software. Hillsdale, NJ: Erlbaum. 
Jonassen, D. H. (2000). Towards a design theory of problem solving. Educational Technology Research and Development, 48(4), 63-85. Retrieve from http://www.springerlink.com.proxy2.library.uiuc.edu/content/tnk3716r532x0827/f ulltext.pdf

Jonassen, D., Strobel, J., \& Lee, C. B. (2006). Everyday problem solving in engineering: lessons for engineering educators. Journal of Engineering Education, 95(2), 139151.

Katehi, L., Pearson, G., Feder, M. A., Committee on K-12 Engineering Education., National Academy of Engineering., \& National Research Council (U.S.). (2009). Engineering in K-12 education: Understanding the status and improving the prospects. Washington, DC: National Academies Press.

Kan, J. W. T. \& Gero, J. S. (2009). Learning to collaborate during team designing: Some preliminary results from measurement-based tools. In A. Chakrabarti (Ed), Research into Design, Research Publications, India, 560-567.

Kan, J. W., \& Gero, J. S. (2010). Exploring quantitative methods to study design behavior in collaborative virtual workspaces. Paper presented at the New Frontiers: CAADRIA 2010, Hong Kong, China.

Kavakli, M., \& Gero, J. S. (2002). The structure of concurrent cognitive actions: a case study on novice and expert designers* 1. Design Studies, 23(1), 25-40.

Kirschenman, M. D., \& Brenner, B. (2011). Civil Engineering Design as the Central Theme in Civil Engineering Education Curriculum. Leadership \& Management In Engineering, 11(1), 69-71. doi:10.1061/(ASCE)LM.1943-5630.0000101

Kolfschoten, G. L., \& de Vreede, G.-J. (January 01, 2009). A design approach for collaboration processes: A multimethod design science study in collaboration engineering. Journal of Management Information Systems, 26, (1), 225-256.

Kusiak, A., \& Larson, N. (1995). Decomposition and representation methods in mechanical design. Journal of Vibration and Acoustics, 117, 17-24.

Lammi, M. D. (2011). Characterizing high school students' systems thinking in engineering design through the function-behavior-structure (FBS) 
framework. Utah State University. ProQuest Dissertations and Theses, Retrieved from http://search.proquest.com/docview/854330953?accountid=14761

Lammi, M. \& Becker, K. (2013). Engineering design thinking. Journal of Technology Education, 24(2), 55-77.

Leighton, J. P. (2004). Avoiding misconception, misuse, and missed opportunities: The collection of verbal reports on educational achievement testing. Educational Measurement: Issues and Practice, 23, 6-15.

Lewis, S., Harris, R., \& Cox, B. (2007). Engineering a better workplace: A diversity guide for the engineering profession. Barton, Australia: A.C.T. Engineers.

Lichtman, M. (2006). Qualitative research in education: A user's guide. Thousand Oaks, CA: Sage.

Liikkanen, L. A., \& Perttula, M. (2009). Exploring problem decomposition in conceptual design among novice designers. Design Studies, 30(1), 38-59. doi:10.1016/j.destud.2008.07.003

Marin, J. A., Armstrong, J. E. \& Kays, J. L. (1999). Elements of an optimal capstone design experience. Journal of Engineering Education, 88, 19-22. doi: 10.1002/j.2168-9830.1999.tb00405.x

Marom, S., Meir R., Braun, E., Gal, A., Kermany, E., \& Eytan, D. (2009). On the precarious path of reverse neuro-engineering. Frontiers in Computational Neuroscience. 3, 5. doi: 10.3389/neuro.10.005.2009.

Marzano, R. J., Brandt, R. S., Hughes, C. S., Jones, B. F., Presseisen, B. Z., Rankin, S. C., \& Suhor, C. (1988). Dimensions of thinking: A framework for curriculum and instruction. Alexandria, VA: Association for Supervision and Curriculum Development.

Mawson, B. (2003). Beyond 'The Design Process': An alternative pedagogy for technology education. International Journal of Technology and Design Education, 13(2), 117-128. 
McCracken, W. M. (1997). Portfolio assessment in design education, FIE, Atlanta, Georgia. http://www.cc.gatech.edu/edutech/dew/papers/McCrackenPos.html.

McDonnell, J., \& Lloyd, P. (Eds.). (2007). DTRS 7: Design meeting protocols. London, UK: University of the Arts.

McMasters, J. H. (2004). Influencing engineering education: One (aerospace) industry perspective. International Journal of Engineering Education, 20(3), 353-371.

Meier, R. L., Williams, M. R., \& Humphreys, M. A. (July, 2000). Refocusing our efforts: assessing non-technical competency gaps. Journal of Engineering Education, $377-385$.

Mendelsahn, R. (January 01, 1998). Teamwork - the key to productivity. Journal of Management in Engineering, 14, 1, 22.

Mentzer, N., \& Becker, K. (2010). Academic preparedness as a predictor of achievement in an engineering design challenge. Journal of Technology Education, 22(1), 2242.

Moore, D. J., \& Voltmer, D. R. (January 01, 2003). Curriculum for an engineering renaissance. IEEE Transactions on Education, 46, 452-455.

Moroney, W. F., Konz, S., \& Green, P. A. (2008). Providing the team experience to human factors and ergonomics students [online]. Retrieved from: http://www.umich.edu/ driving/publications/ProvidingTeamExperience.pdf.

Mulet, E., \& Vidal, R. (2008). Heuristic guidelines to support conceptual design. Research in Engineering Design, 19(2/3), 101-112. doi:10.1007/s00163-0080050-5

Nair, C. S., Mertova, P., \& Patil, A. (July 22, 2009). Re-engineering graduate skills - A case study. European Journal of Engineering Education, 34(2), 131-139.

Olson, G. M., \& Olson, J. S. (2000). Distance matters. Human-Computer Interaction, 15, 130-178. 
O'Neil, C. E., \& Lima, M. (2003). Service-learning in agricultural instruction: A guide for implementing real-world, hands-on, community based teaching and learning. NACTA Journal, 2(1), 36-41.

Ormerod, T. C., \& Ridgway, J. (1999) Developing task design guides through cognitive studies of expertise. In European Conference on Cognitive Science (ECCS99), pp. 401-410. UNSPECIFIED, Sienna, Italy.

Pahl, G., Beitz, W., Schulz, H., Jarecki, U., Wallace, K., \& Blessing, L. T. M. (2007). Engineering design: A systematic approach. New York, NY: Springer.

Patil, A. S. (2005). The global engineering criteria for the development of a global engineering profession. World Transaction on Engineering Education, 4(1), 4952.

Radcliffe, D. F., \& Lee, T. Y. (1989). Design methods used by undergraduate engineering students. Design Studies, 10(4), 199-207.

Rau, W., \& Heyl, B. S. (1990). Humanizing the college classroom: Collaborative learning and social organization among students. Teaching Sociology, 18(2), 141155.

Romero, N. Y. D., Slater, P., \& DeCristofano, C. (2006). Design challenges are "ellementary". Science and Children, 43(4), 34-37.

Roselli, R. J., \& Brophy, S. P. (2006). Effectiveness of challenge-based instruction in biomechanics. Journal of Engineering Education, 95(4), 311-324.

Sageev, P., \& Romanowski, C. J. (October, 2001). A message from recent engineering graduates: results of a survey on technical communication skills. Journal of Engineering Education, 685-692.

Schierwagen, A. (2012). On reverse engineering in the cognitive and brain sciences. Natural Computing, 11(1), 141-150. doi:10.1007/s11047-012-9306-0

Schloss, P. J., \& Smith, M. A. (1999). Conducting research. Upper Saddle River, NJ: Prentice Hall. 
Scott, G., \& Yates, K.W. (2002). Using successful graduates to improve the quality of undergraduate engineering programmes. European Journal of Engineering Education, 2002(24), 363-378.

Sheppard, S. D. (2003). A description of engineering: An essential backdrop for interpreting engineering education. Proceedings (CD), Mudd Design Workshop IV. Claremont, CA: Harvey Mudd College.

Sheppard, S. D., Macatangay, K., Colby, A., \& Sullivan, W. M. (2009). Educating engineers: Designing for the future of the field. San Francisco, CA: Jossey-Bass.

Simon, H. A. (1996). The sciences of the artificial (3rd ed.). Cambridge, MA: MIT Press.

Smith, K. A. (2008). Strategies for developing engineering student's teamwork and project management skills [online]. Retrieved from: http://faculty.kfupm.edu.sa/COE/elrabaa/rich\%20text/general\%20presentations/2 0565.pdf.

Soloway, E., Guzdial, M., \& Hay, K. (1994). Learner-centered design. Interactions, 1, 36-48.

Todd, R. H., \& Magleby, S. P. (2004). Evaluation and Rewards for Faculty Involved in Engineering Design Education. International Journal of Engineering Education, 20(3), 333-340.

Vermaas, P. E., \& Dorst, C. H. (2007). On the conceptual framework of John Gero's fbsmodel and the prescriptive aims of design methodology. Design Studies, 28(2), 133-157.

Vezzetti, E. E. (2009). Adaptive sampling plan design methodology for reverse engineering acquisition. International Journal of Advanced Manufacturing Technology, 42(7/8), 780-792. doi:10.1007/s00170-008-1625-z

Vincenti, W. G. (1990): What engineers know and how they know it, analytical studies from aeronautical history. Baltimore, MA: Johns Hopkins University Press.

Williams, C. B., Gero, J., Lee, Y., \& Paretti, M. (2011). Exploring the effect of design education on the design cognition of mechanical engineering students. 
Proceedings of the ASME 2011 International Design Engineering Technical Conference \& Computer and Information in Engineering Conference, pp. 1-8. Washington, DC.

Yaeger, P. M. (2002). Innovations and outcomes in engineering education: Active learning in dynamics classes. Unpublished doctoral dissertation, Pennsylvania State University, State College.

Yang, M.C. (2005). A study of prototypes, design activity, and design outcome. Design Studies, 26(6), 649-669.

Zolin, R., Hinds, P. J., Fruchter, R., \& Levitt, R. E. (2004). Interpersonal trust in crossfunctional, geographically distributed work: A longitudinal study. Information and Organization, 14, 1-26. 
APPENDICES 
Appendix A

Visual Map of the Literature 


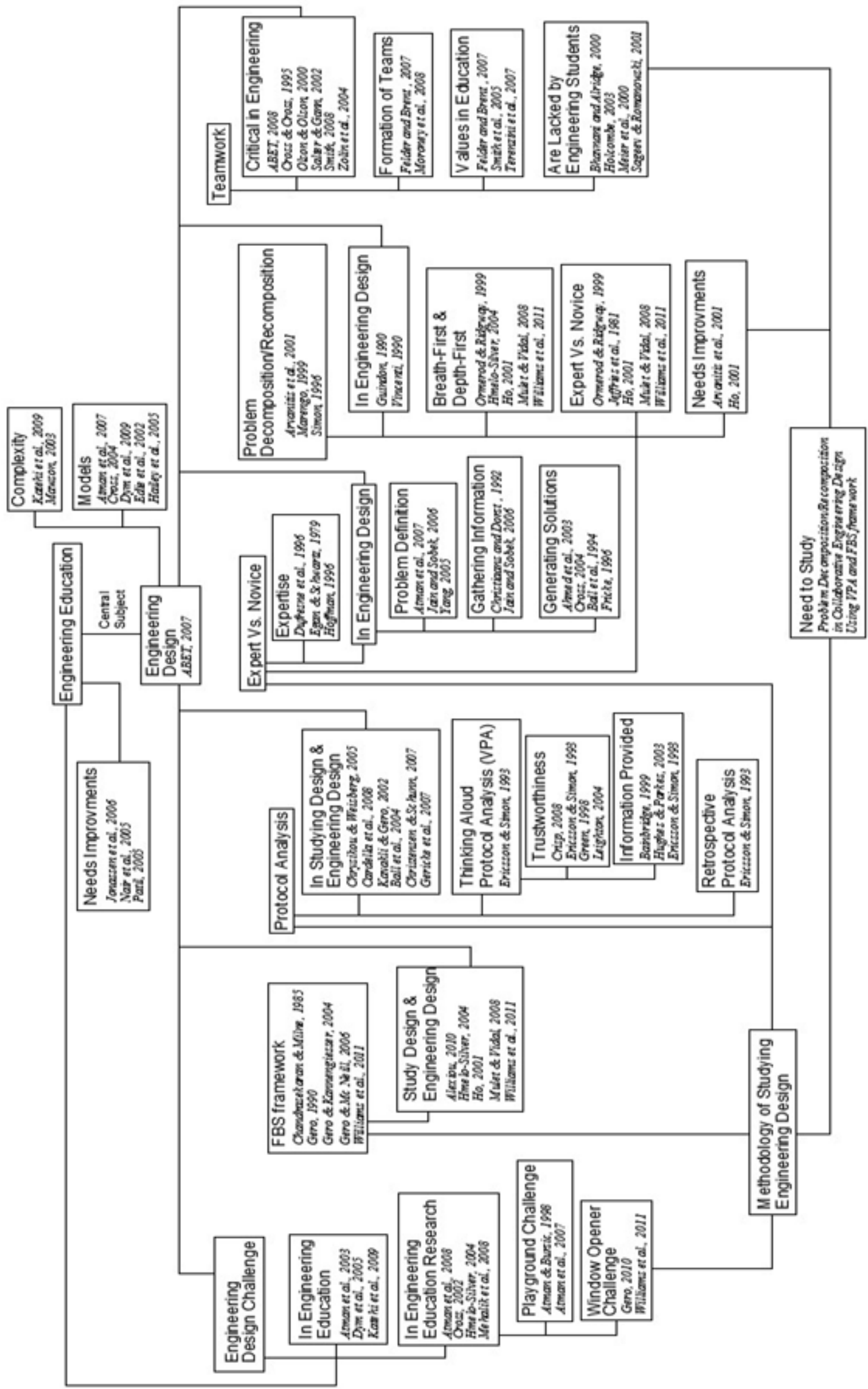


Appendix B

Double-Hung Window Opener Design Challenge 


\section{Double-Hung Window Opener}

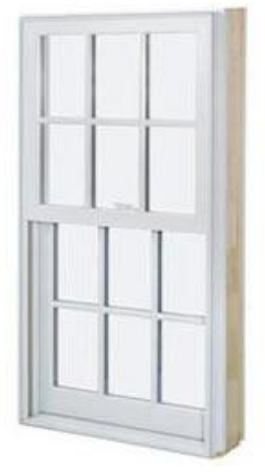

Your design team has been approached by a local nursing home to design a new product to assist its elderly residents. The nursing home administrators have noticed that changes in humidity during the summer months cause the windows of the 65-year old building to "stick," thus requiring significant amounts of force to raise and lower the window panes. The force required to adjust the windows is often much too large for the nursing home tenants, making it very difficult for them to regulate their room temperature.

Your team has been tasked with designing a device that will assist the elderly tenants with raising and lowering the building's windows. You will produce a complete engineering design solution for the client. Someone should be able to build the device from your solution without any questions. Since each window is not guaranteed to be located near an electrical socket, this device should not rely on electric power.

The building's windows are double-hung (as seen in the figure above). The double-hung window consists of an upper and lower sash that slide vertically in separate grooves in the side jambs. This type of window provides a maximum face opening for ventilation of one-half the total window area. Each sash is provided with springs, balances, or compression weather stripping to hold it in place in any location.

Your team has identified the following websites as potential sources of useful information: "Double Hung Window Construction": http://www.oldhouseweb.com/how-to-advice/doublehung-window-construction.shtml

"Double Hung Windows - Everything You Need to Know" (1 min. 34 sec.): http://www.youtube.com/watch?v=xW7OMHYI4kY

American Disabilities Act (ADA) information: http://www.ada.gov/

ADA Accessibility Guidelines for Buildings and Facilities (ADAAG): http://www.accessboard.gov/adaag/html/adaag.htm

\section{References:}

Lammi, M. D. (2011). Characterizing high school students' systems thinking in engineering design through the function-behavior-structure (FBS) framework. Utah State University). ProQuest Dissertations and Theses, Retrieved from http://search.proquest.com/docview/854330953?accountid=14761 http://www.windowquoteusa.com/Window-types/Double-Hungs.html 
Appendix C

Interview Questions 


\section{Guiding Questions:}

1. How did you define the problem?

2. How did you decide what information to get?

3. How did you develop or come across different ideas (solutions)?

4. How did you know which ideas would work and which would not work?

5. Why and how did you choose your final idea or plan?

6. Is there anything else you needed or wanted that would have helped you?

7. Did you tackle the problem as a whole or decompose it into several sub-problems? If you decomposed it, why did you choose it over the other one?

8. What difficulties did you meet in solving the problem? 
Appendix D

Anonymous Demographic Questionnaire for Students 
Please answer each of the questions as honestly as you can. Your responses are anonymous.

1. What is your gender?
a. Female
b. Male

2. What ethnicity do you primarily identify with?
a. African American
b. Alaskan Native
c. American Indian
d. Asian
e. Filipino
f. Hispanic
g. Pacific Islander
h. White
i. Other

3. What is your age?

4. What is your college grade point average (GPA)?

5. What is your year in school as of today?

6. What is the highest level of education obtained by either of your parents/guardians?
A. Some High School
B. High School/GED
C. Some College
D. Associates Degree
E. Bachelors Degree
F. Masters Degree
G. Doctoral Degree
H. Other, Please Specify: 
Appendix E

Anonymous Demographic Questionnaire for Engineers 
Please answer each of the questions as honestly as you can. Your responses are anonymous.

1. What is your gender?

c. Female

d. Male

2. What ethnicity do you primarily identify with?

j. African American

k. Alaskan Native

1. American Indian

m. Asian

n. Filipino

o. Hispanic

p. Pacific Islander

q. White

r. Other

3. What is your age?

4. How many years have you worked as an engineer?

5. What is the highest level of education obtained by either of your parents/guardians?
A. Some High School
B. High School/GED
C. Some College
D. Associates Degree
E. Bachelors Degree
F. Masters Degree
G. Doctoral Degree
H. Other, Please Specify: 
Appendix F

Informed Consent Form for Students 


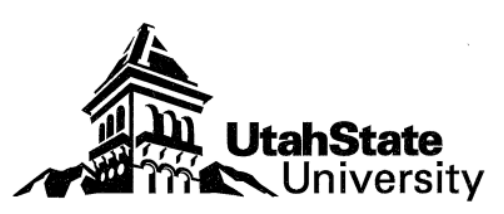

Department of Engineering Education

4160 Old Main Hill

Logan UT 84322-4160

Telephone: (435) 797-7020

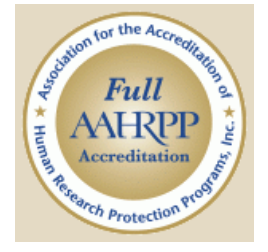

138

INFORMED CONSENT

Expert vs. Novice: Problem Decomposition/Recomposition in Engineering Design

Introduction/ Purpose Dr. Kurt Becker in the Department of Engineering Education at Utah State University is conducting a research study to find out more about engineering design. You have been asked to take part because the target population of the study includes college engineering freshmen, senior, and experienced engineers. There will be approximately 50 total participants in this research. Ting Song will be the graduate student researcher involved in this study.

Procedures If you agree to take part in this research study, you will work with another participant to complete an engineering design challenge. Before the design challenge, you will fill out an anonymous demographic questionnaire. You will have an hour to work with your partner to finish the challenge, and all of your design activities will be video recorded. After completing the design challenge, you will participate in an interview that will ask questions about how you framed the problem, generated alternative solutions, reached agreements, and used strategies. The whole process will last no more than three hours. After this participation, there will be no follow-up.

Alternative Procedures Instead of participating in this pilot study, an alternative for you to consider would be: participate this study in the future.

$\underline{\text { Risks }}$ There are no foreseen risks that might occur in this study.

Research-Related Injuries There is minimal risk in participating in this study. Your participation will not impact your class grade.

Benefits There are no direct benefits to the participants, but this study will contribute to the research of engineering design. The direct significance of the study include: quantitatively characterizing the design thinking of both engineering students and expert engineers from a design cognition perspective; measuring differences between the design cognition of engineering students and expert engineers; adding to the knowledge of the development of design thinking across education and experience; and proposing educational interventions that narrow the differences between students and experts. Equally important, by applying a cognitively based measurement technique for studying design thinking, this study will contribute to the development of quantitative measurements of the effects of educational interventions and of experience. In addition, the findings of this study will help engineering educators improve the curriculum of engineering design. New teaching strategies may be developed in order to teach students expert-like skills, which will contribute to the improvement of college engineering education. 


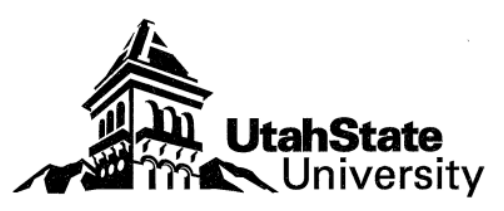

Department of Engineering Education

4160 Old Main Hill

Logan UT 84322-4160

Telephone: (435) 797-7020

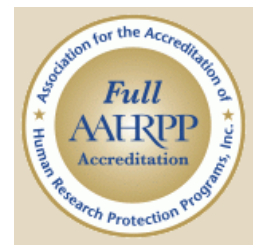

139

INFORMED CONSENT

Expert vs. Novice: Problem Decomposition/Recomposition in Engineering Design

Explanation \& offer to answer questions Ting Song (Research Assistant) has explained this research study to you and answered your questions. If you have other questions or research-related problems, you may reach (PI) Kurt Becker at (435) 7972076 or kurt.becker@usu.edu.

Payment/Compensation You will receive a $\$ 20$ cash honorarium for your participation in this study. (**Note: If you will receive payments, gift cards or similar items of value for participating in this research, the Internal Revenue Service (IRS) has determined that if the amount you get from this study, plus any prior amounts you have received from participating in research studies at USU since January of this year, total $\$ 600$ or more, USU must report this income to the federal government. If you are a USU employee, any payment you receive from this study will be included in your regular payroll).

\section{Voluntary nature of participation and right to withdraw without consequence}

Participation in the research is entirely voluntary. You may refuse to participate or withdraw at any time without consequence or loss of benefits. You may be withdrawn from this study without your consent by the investigator.

Confidentiality Research records will be kept confidential, consistent with federal and state regulations. Only the investigator will have access to the data which will be kept in a locked file cabinet or on a password protected computer in a locked room. To protect your privacy, personal, identifiable information will be removed from study documents and replaced with a study identifier. Identifying information will be stored separately from data and will be kept. Identifying information will be destroyed six months after the data are collected.

IRB Approval Statement The Institutional Review Board for the protection of human participants at Utah State University has approved this research study. If you have any questions or concerns about your rights or a research-related injury and would like to contact someone other than the research team, you may contact the IRB Administrator at (435) 797-0567 or email irb@ usu.edu to obtain information or to offer input.

Copy of consent You have been given two copies of this Informed Consent. Please sign both copies and keep one copy for your files.

Investigator Statement "I certify that the research study has been explained to the individual, by me or my research staff, and that the individual understands the nature and purpose, the possible risks and benefits associated with taking part in this research study. Any questions that have been raised have been answered." 


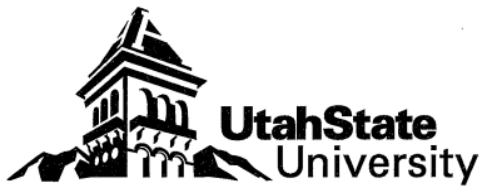

Department of Engineering Education

4160 Old Main Hill

Logan UT 84322-4160

Telephone: (435) 797-7020

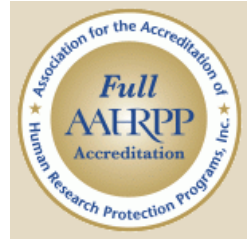

INFORMED CONSENT

Expert vs. Novice: Problem Decomposition/Recomposition in Engineering Design

Signature of Researcher(s)

Kurt Becker

Principal Investigator

435-797-2076

kurt.becker@usu.edu
Ting Song

Student Researcher (or Co-PI)

217-377-6933

ting.song@usu.edu

Signature of Participant By signing below, I agree to participate.

Participant's signature

Date 
Appendix G

Informed Consent Form for Engineers 


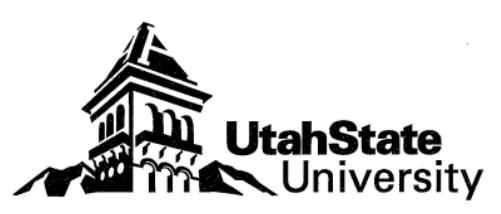

Department of Engineering Education

4160 Old Main Hill

Logan UT 84322-4160

Telephone: (435) 797-7020

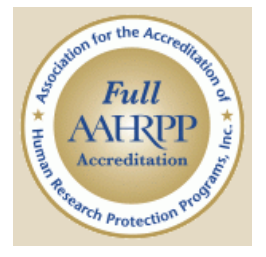

\section{Expert vs. Novice: Problem Decomposition/Recomposition in Engineering Design}

Introduction/ Purpose Dr. Kurt Becker in the Department of Engineering Education at Utah State University is conducting a research study to find out more about engineering design. You have been asked to take part because the target population of the study includes college engineering freshmen, senior, and experienced engineers. There will be approximately 50 total participants in this research. Ting Song will be the graduate student researcher involved in this study.

Procedures If you agree to take part in this research study, you will work with another participant to complete an engineering design challenge. Before the design challenge, you will fill out an anonymous demographic questionnaire. You will have an hour to work with your partner to finish the challenge, and all of your design activities will be video recorded. After completing the design challenge, you will participate in an interview that will ask questions about how you framed the problem, generated alternative solutions, reached agreements, and used strategies. The whole process will last no more than three hours. After this participation, there will be no follow-up.

Alternative Procedures Instead of participating in this pilot study, an alternative for you to consider would be: participate this study in the future.

$\underline{\text { Risks }}$ There are no foreseen risks that might occur in this study.

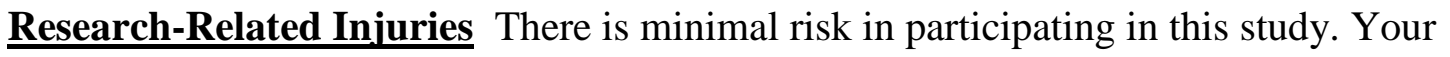
participation will not impact your class grade.

Benefits There are no direct benefits to the participants, but this study will contribute to the research of engineering design. The direct significance of the study include:

quantitatively characterizing the design thinking of both engineering students and expert engineers from a design cognition perspective; measuring differences between the design cognition of engineering students and expert engineers; adding to the knowledge of the development of design thinking across education and experience; and proposing educational interventions that narrow the differences between students and experts. Equally important, by applying a cognitively based measurement technique for studying design thinking, this study will contribute to the development of quantitative measurements of the effects of educational interventions and of experience. In addition, the findings of this study will help engineering educators improve the curriculum of engineering design. New teaching strategies may be developed in order to teach students expert-like skills, which will contribute to the improvement of college engineering education. 


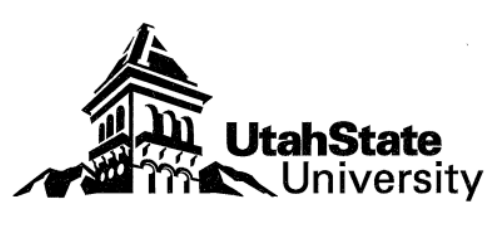

Department of Engineering Education

4160 Old Main Hill

Logan UT 84322-4160

Telephone: (435) 797-7020

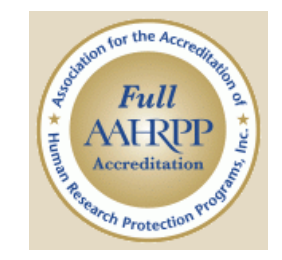

\section{Expert vs. Novice: Problem Decomposition/Recomposition in Engineering Design}

Explanation \& offer to answer questions Ting Song (Research Assistant) has explained this research study to you and answered your questions. If you have other questions or research-related problems, you may reach (PI) Kurt Becker at (435) 7972076 or kurt.becker@usu.edu.

Payment/Compensation You will receive a $\$ 50$ cash honorarium for your participation in this study. (**Note: If you will receive payments, gift cards or similar items of value for participating in this research, the Internal Revenue Service (IRS) has determined that if the amount you get from this study, plus any prior amounts you have received from participating in research studies at USU since January of this year, total $\$ 600$ or more, USU must report this income to the federal government. If you are a USU employee, any payment you receive from this study will be included in your regular payroll).

\section{Voluntary nature of participation and right to withdraw without consequence}

Participation in the research is entirely voluntary. You may refuse to participate or withdraw at any time without consequence or loss of benefits. You may be withdrawn from this study without your consent by the investigator.

Confidentiality Research records will be kept confidential, consistent with federal and state regulations. Only the investigator will have access to the data which will be kept in a locked file cabinet or on a password protected computer in a locked room. To protect your privacy, personal, identifiable information will be removed from study documents and replaced with a study identifier. Identifying information will be stored separately from data and will be kept. Identifying information will be destroyed six months after the data are collected.

IRB Approval Statement The Institutional Review Board for the protection of human participants at Utah State University has approved this research study. If you have any questions or concerns about your rights or a research-related injury and would like to contact someone other than the research team, you may contact the IRB Administrator at (435) 797-0567 or email irb@usu.edu to obtain information or to offer input.

Copy of consent You have been given two copies of this Informed Consent. Please sign both copies and keep one copy for your files.

Investigator Statement "I certify that the research study has been explained to the individual, by me or my research staff, and that the individual understands the nature and purpose, the possible risks and benefits associated with taking part in this research study. Any questions that have been raised have been answered." 


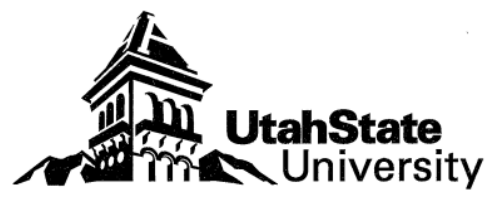

Department of Engineering Education

4160 Old Main Hill

Logan UT 84322-4160

Telephone: (435) 797-7020

\section{INFORMED CONSENT}

Expert vs. Novice: Problem Decomposition/Recomposition in Engineering Design

\section{Signature of Researcher(s)}

Kurt Becker

Principal Investigator

435-797-2076

kurt.becker@usu.edu
Ting Song

Student Researcher (or Co-PI)

217-377-6933

ting.song@usu.edu

Signature of Participant By signing below, I agree to participate.

Participant's signature

Date 
VITAE 


\section{Ting Song}

Department of Engineering Education

Utah State University, Logan, UT-84322-4160

Cell phone: (435)764-4393

E-mail: songitaly@gmail.com

Web: http://eed.usu.edu

\section{EDUCATION}

PhD, Engineering Education

May 2014

Utah State University, Logan, Utah, USA

GPA 3.90

Thesis: "Expert vs. Novice: Problem Decomposition/Recomposition in Engineering

Design" Advisor: Dr. K. Becker

M. S., Engineering and Technology Education

December 2010

Utah State University, Logan, Utah, USA

GPA 3.87

"A curriculum unit to introduce engineering to junior high school students" Advisor: Dr.

K. Becker

B. E., Environmental Engineering

July

2006Tianjin University, Tianjin, China

\section{RESEARCH INTERESTS}

Engineering Education

Curriculum development

Project-based learning

Design thinking

Engineering design

\section{RESEARCH AND TEACHING EXPERIENCE}

Graduate Student/Research Assistant, Department of Engineering 2009-present Education, Utah State University

Logan, UT

- Developed Curriculum on middle school level

- Wrote NSF proposals in collaboration with Professor K. Becker and Professor E. Reeve

- Wrote National Center for Engineering and Technology Education (NCETE) Annual Report in collaboration with Professor K. Becker

- Developed surveys and interviews

- Recruited undergraduate students to participate in studies

- Analyzed quantitative data using SPSS

- Analyzed qualitative data using Constant Comparative Method (CCM) 
- Attended a training session from Dr. John Gero for coding Function-BehaviorStructure (FBS) frame work at George Mason University

- Worked as a mentor in an REU project funded by NSF

Teaching Assistant of Computer Engineering Drafting

2011-2013

Department of Engineering Education, Utah State University

Logan, UT

- Delivered lectures to undergraduate students

- Graded homework

- Answered questions in lab sessions

- Managed online Canvas system

- Delivered examinations

- Managed 3D printer

\section{WORK EXPERIENCE}

Civil Engineer

2006-2008

Xiangtai City Planning Design Co.

Tianjin, China

- Used AutoCAD to design civil pipelines

- Worked with clients to assess needs

- Worked with government departments to negotiate policies

- Participated in design drainage projects as the major designer, including:

$\checkmark \quad$ Nankai the Third Avenue (2008 Beijing Olympic Games Associate Projects in Tianjin)

$\checkmark$ Beijing Olympics Soccer Court in Tianjin (2008 Beijing Olympic Games Associate Projects in Tianjin)

$\checkmark$ Nanjing Avenue (2008 Beijing Olympic Games Associate Projects in Tianjin)

$\checkmark$ West Railway Station Street

$\checkmark$ Multiple roads, streets, and avenues

\section{PROPOSAL DEVELOPMENT}

National Science Foundation (Fall 2010). Worked with Dr. Edward Reeve to develop TUES proposal (10-544).

National Science Foundation (Fall 2011). Worked with Dr. Kurt Becker to develop DRK12 proposal (10-1340).

\section{CURRICULUM DESIGN}

Making Recycled Paper - introduce engineering to middle school students (Fall 2010).

\section{PRESENTATIONS}

"Investigating the Engineering Design Process: Novices vs. Experts" 2012 Frontiers in Education Conference (October, 2012)

"Work in Progress: Investigating the Engineering Design Process", Department Research Seminar (February, 2013) 
“Engineering Design: Novices vs. Experts”, Department Research Seminar (March, 2014)

\section{PUBLICATIONS}

Making Recycled paper: An Engineering Design Challenge in Technology and Engineering Teacher (October 2013, 30-34).

Expert vs. Novice: Problem Decomposition/Recomposition in Engineering Design WEEF (World Engineering Education Forum) (Abstract accepted in April, 2014).

\section{RELATED COURSES}

- Communication, Instruction, and the Learning Process

- Interactive Multi-Media Production

- The Role of Cognition in Engineering and Technology Education

- Teaching-Learning Foundations in Education

- Historical, Social, and Cultural Foundations of Education

- Designing and Interpreting Measurements for Assessing Student Learning

- Research and Evaluation in Instructional Technology

- Administration and Organization in Education

- Occupational Analysis and Curriculum Development

- Internationalizing Institutions of Higher Education

- Social Foundations of Education

- Finance and Grant Writing

- Design of Experiments

- Research Design and Analysis

- Qualitative Methods

\section{LEADERSHIP}

Graduate Student Senator, Department of Engineering Education, USU, 2010-2011

Vice President of Finance, ASEE at Utah State University, USU, 2013-present 